\title{
ISM properties in low-metallicity environments
}

\section{Mid-infrared spectra of dwarf galaxies ${ }^{\star}$}

\author{
S. C. Madden ${ }^{1}$, F. Galliano ${ }^{1,2}$, A. P. Jones ${ }^{3}$, and M. Sauvage ${ }^{1}$ \\ 1 Service d'Astrophysique, CEA/Saclay, l'Orme des Merisiers, 91191 Gif-sur-Yvette, France \\ e-mail: smadden@cea.fr \\ 2 Observational Cosmology Lab., Code 665, NASA Goddard Space Flight Center, Greenbelt MD 20771, USA \\ 3 Institut d'Astrophysique Spatiale (CNRS), Université de Paris XI, 91405 Orsay, France
}

Received 22 July 2005 / Accepted 27 September 2005

\section{ABSTRACT}

We present new ISOCAM mid-infrared spectra of three starbursting nearby dwarf galaxies, NGC 1569, NGC 1140 and II Zw 40 and the 30 Dor region of the LMC and explore the properties of the ISM in low-metallicity environments, also using additional sources from the literature. We analyse the various components of the ISM probed by the mid-infrared observations and compare them with other Galactic and extragalactic objects. The MIR spectra of the low-metallicity starburst sources are dominated by the [Ne III] $\lambda 15.56 \mu \mathrm{m}$ and [S IV] $\lambda 10.51 \mu \mathrm{m}$ lines, as well as a steeply rising dust continuum. PAH bands are generaly faint, both locally and averaged over the full galaxy, in stark contrast to dustier starburst galaxies, where the PAH features are very prominant and even dominate on global scales. The hardness of the modeled interstellar radiation fields for the dwarf galaxies increases as the presence of PAH band emission becomes less pronounced. The [Ne III]/[Ne II] ratios averaged over the full galaxy are strikingly high, often $>10$. Thus, the hard radiation fields are pronounced and pervasive. We find a prominent correlation between the PAHs/VSGs and the $[\mathrm{Ne}$ III]/[Ne II] ratios for a wide range of objects, including the low metallicity galaxies as well as Galactic H II regions and other metal-rich galaxies. This effect is consistent with the hardness of the interstellar radiation field playing a major role in the destruction of PAHs in the low metallicity ISM. We see a PAHs/VSGs and metallicity correlation, also found by Engelbracht et al. $(2005, \mathrm{ApJ}, 628,29)$ for a larger survey. Combined effects of metallicity and radiation field seem to be playing important roles in the observed behavior of PAHs in the low metallicity systems.

Key words. galaxies: dwarf - galaxies: starburst - ISM: general - galaxies: ISM

\section{Introduction}

Understanding the interplay between star formation and the interstellar medium (ISM) in low-metallicity environments, perhaps characteristic of primordial galaxies, can be approached through observations of the local universe dwarf galaxies. Much of what we have gleaned about the state of the ISM and star formation properties in dwarf galaxies has been derived historically, from optical photons. We have access to a vast Infrared Space Observatory (ISO) mid-infrared (MIR) database, and more recently, Spitzer observations, from which new thoughts on the nature of the ISM of dwarf galaxies are emerging. For example, the amount of dust that is actually present in dwarf galaxies is being called into question in several cases (Hunt et al. 2001; Vacca et al. 2002; Plante \& Sauvage 2002; Lisenfeld et al. 2002; Galliano et al. 2003, 2005), as a result of the modeling of the dust emission spectral energy

* Based on observations with ISO, an ESA project with instruments funded by ESA Member States (especially the PI countries: France, Germany, The Netherlands and the United Kingdom) and with the participation of ISAS and NASA. distribution (SED). The myth that the effects of dust can be ignored or treated lightly in dwarf galaxies is on the way to banishment, and opens our eyes to the possibility of optically hidden, embedded star formation activity in these low metallicity systems.

Until recently, our knowledge of the dust emission properties of dwarf galaxies was dependent primarily on 4 IRAS bands (e.g. Hunter et al. 1989; Melisse \& Israel 1994; Sauvage $\&$ Thuan 1994). ISO provided the sensitivity to begin the exploration of the details of the MIR wavelength regime, where a wide variety of physical components of the ISM can be traced. The MIR camera on board ISO, ISOCAM (Cesarsky et al. 1996a), with its spectro-imaging capability, has proven to be invaluable in probing the various components of the MIR regime of a number of low-metallicity dwarf galaxies. ISOCAM first demonstrated the widely-varying MIR characteristics of low metallicity starburst galaxies (Madden 2000), which differ remarkably from those of the more metal-rich starburst galaxies (e.g. Rigopoulou et al. 1999; Laurent et al. 2000; Sturm et al. 2000, 2002; Förster Schreiber et al. 2003). The improved sensitivity of the Spitzer telescope will provide a means to delve 
Table 1. Sources used in this study. The dates indicate when the observations were made by ISO; the RA and Dec are the coordinates of the center of the field. References for the distances and metallicity values are the following: ${ }^{a}$ Israel (1988); ${ }^{b}$ Kobulnicky \& Skillman (1997); ${ }^{c}$ Thuan \& Martin (1981); ${ }^{d}$ Brinks \& Klein (1988); ${ }^{e}$ Guseva et al. (2000); ${ }^{f}$ Hunter et al. (1994); ${ }^{g}$ Heckman et al. (1998); ${ }^{i}$ Calzetti (1997); ${ }^{j}$ Freedman et al. (2001); ${ }^{k}$ Kobulnicky et al. (1997); ${ }^{l}$ van den Bergh (1999); ${ }^{m}$ Dufour et al. (1982). For comparison, the solar metallicity is $12+\log \mathrm{O} / \mathrm{H}=8.83$ (Grevesse \& Sauval 1998).

\begin{tabular}{|c|c|c|c|c|c|c|}
\hline Source & Date & RA (J2000) & $\overline{\text { Dec (J2000) }}$ & Distance & $12+\log (\mathrm{O} / \mathrm{H})$ & MIR references \\
\hline NGC 1569 & Mar. 1998 & $04^{\mathrm{h}} 30^{\mathrm{m}} 49^{\mathrm{s}} .1$ & $64^{\circ} 50^{\prime} 52.8^{\prime \prime}$ & $2.2 \mathrm{Mpc}^{a}$ & $8.19^{b}$ & this study \\
\hline II Zw 40 & Oct. 1997 & $05^{\mathrm{h}} 55^{\mathrm{m}} 42^{\mathrm{s}} .7$ & $3^{\circ} 23^{\prime} 29.5^{\prime \prime}$ & $10 \mathrm{Mpc}^{c, d}$ & $8.09^{e}$ & this study \\
\hline NGC 1140 & Feb. 1998 & $2^{\mathrm{h}} 54^{\mathrm{m}} 33^{\mathrm{s}} .5$ & $-10^{\circ} 01^{\prime} 44.0^{\prime \prime}$ & $23 \mathrm{Mpc}^{f, g, h}$ & $8.44^{i}$ & this study \\
\hline NGC 5253 & Jan. 1997 & $13^{\mathrm{h}} 39^{\mathrm{m}} 56^{\mathrm{s}} .0$ & $-31^{\circ} 38^{\prime} 29.0^{\prime \prime}$ & $3.25 \mathrm{Mpc}^{j}$ & $8.16^{k}$ & Crowther et al. (1999) \\
\hline SMC N 66 & Sep. 1996 & $0^{\mathrm{h}} 59^{\mathrm{m}} 02^{\mathrm{s}} .0$ & $-72^{\circ} 10^{\prime} 36.0^{\prime \prime}$ & $60 \mathrm{kpc}^{l}$ & $8.04^{m}$ & Contursi et al. (2000) \\
\hline SMC B\#1 & Jul. 1996 & $0^{\mathrm{h}} 45^{\mathrm{m}} 33^{\mathrm{s}} .0$ & $-73^{\circ} 18^{\prime} 46.0^{\prime \prime}$ & $60 \mathrm{kpc}^{l}$ & $8.04^{m}$ & Reach et al. (2000) \\
\hline LMC 30 Dor & Oct. 1997 & $5^{\mathrm{h}} 38^{\mathrm{m}} 34^{\mathrm{s}} .0$ & $-69^{\circ} 05^{\prime} 57.0^{\prime \prime}$ & $50 \mathrm{kpc}^{l}$ & $8.37^{m}$ & this study \\
\hline
\end{tabular}

even deeper into the detailed MIR properties of dwarf galaxies (Engelbracht et al. 2005). The availability of the details of the MIR wavelength regime launched the study of the MIR to millimetre $(\mathrm{mm})$ SEDs of dwarf galaxies, from which the nature of the dust properties in galaxies can be more accurately modeled. The spectral properties of the MIR SED are particularly important factors in constraining dust models for the MIR to millimetre (mm) SEDs of dwarf galaxies (Plante \& Sauvage 2002; Takeuchi et al. 2003; Galliano et al. 2003; Vanzi \& Sauvage 2004; Galliano et al. 2005).

Some of the brightest dwarf galaxies have also been valiantly observed from the ground at MIR wavelengths (e.g. Rieke \& Low 1972; Frogel et al. 1982; Roche et al. 1991; Dudley 1999; Vacca et al. 2002; Plante \& Sauvage 2002; Vanzi \& Sauvage 2004). In this paper, we examine the MIR spectra of a sample of low metallicity galaxies, using ISOCAM spectra from $\lambda=5 \mu \mathrm{m}$ to $16 \mu \mathrm{m}$.

Great progress has been made in understanding the MIR interstellar gas and dust characteristics through recent studies of Galactic regions and galaxies with metal-rich ISM (e.g. Helou et al. 2000; Vigroux et al. 2001; Roussel et al. 2001a,b; Dale \& Helou 2002; Lutz et al. 1996; Sturm et al. 2000), and we can now explore the effects of low-metallicity on the dust and gas properties. The MIR regime provides unique advantages for characterising the physical properties of the dust and gas in galaxies, particularly due to the fact that it contains a wide variety of ISM diagnostics that are not heavily affected by extinction. There is a factor of 10 to 50 less extinction over MIR wavelengths compared to optical wavelengths (Mathis 1990). Nebular emission lines from neon, argon and sulfur are present in the ISOCAM range, displaying unique new global properties of star formation activity, which can have some bearing on the low-metallicity environment, directly or indirectly. An important aspect in studying dwarf galaxies in the MIR wavelength range is to discern and understand the variations in the spectral characteristics of the dust and gas emission between the low-metallicity and more metal-rich ISM, perhaps establishing useful tracers for possible primordial conditions in more distant galaxies.

For this study we present new MIR ISOCAM spectra for the 3 dwarf galaxies, NGC 1569, NGC 1140 and II Zw 40, whose full infrared (IR) SEDs have been succesfully modeled by Galliano et al. (2003, 2005), as well as the MIR spectra toward 30 Dor in the LMC. Additionally, we add to our study other low metallicity sources which have previously been presented: NGC 5253 (Crowther et al. 1999); the 1/41 $Z_{\odot}$ galaxy, SBS 0335-052 (Thuan et al. 1999); a quiescent molecular cloud in the SMC, SMC B\#1 (Reach et al. 2000) and N66 in the SMC (Contursi et al. 2000). All of these galaxies possess massive young star clusters and/or super star clusters (SSCs), which represent unusual and relatively rare modes of star formation with stellar surface densities orders of magnitude in excess of normal $\mathrm{H}$ II regions and $\mathrm{OB}$ associations, some of which can be deeply embedded, concealing the precise nature of the star formation. The combination of the relatively high angular resolution and high sensitivity in the MIR using ISOCAM, provides a unique opportunity to study the impact of the environment of SSCs on the nature of the dust. As SSCs have strong stellar winds from the densely packed, rapidly-evolving stars, the presence of dust, as well as gas in the immediate vicinity may not immediately be obvious and may be hindering our view of star formation.

The 7 low metallicity sources discussed in this article range in metallicity from $Z=1 / 41 Z_{\odot}$ to $1 / 2 Z_{\odot}$, and provide a variety of spatial scales, from our neighboring Magellanic Clouds ( $D \simeq 50 \mathrm{kpc}$ to $60 \mathrm{kpc}$ ), to that of our most distant source, NGC $1140(D \simeq 23 \mathrm{Mpc}$; Table 1). Thus, the linear physical scales range from $1.2 \mathrm{pc}$ to $500 \mathrm{pc}$, given the short wavelength spatial resolution $\left(\sim 5^{\prime \prime}\right)$ of ISOCAM on ISO.

The paper is organised as follows: Sect. 2 presents the new ISOCAM CVF observations, Sect. 3 is the spectral analysis, describing how the components of MIR spectra are modeled, isolating the MIR nebular lines, the PAH bands and the continua. Also in Sect. 3, we compare the properties of some of the galaxies with the Galactic H II region/molecular cloud, M 17. Further interpretation of the ionic line ratios, the PAH bands, and the continuum emission are presented in Sect. 4, along with other sources presented here and in the literature. Section 5 ties up the paper with a summary.

\section{Observations and data reduction}

The CVF spectro-images were made with ISOCAM (Cesarsky et al. 1996a) on board the ISO satellite (Kessler et al. 1996), using a $32 \times 32$ detector array with a sampling of $6^{\prime \prime}$ pixel $^{-1}$ for NGC 1569 and NGC 1140, and $3^{\prime \prime}$ pixel $^{-1}$ for II Zw 40, 
giving a camera field of view of $192^{\prime \prime} \times 192^{\prime \prime}$ and $96^{\prime \prime} \times 96^{\prime \prime}$, respectively. The CVF performed spectral imaging from $\lambda=$ $5 \mu \mathrm{m}$ to $16.5 \mu \mathrm{m}$ with one pointing of two CVFs, from $\lambda=5$ to $9.5 \mu \mathrm{m}$ and from $\lambda=9.0$ to $16.5 \mu \mathrm{m}$, with a spectral resolution of $\lambda / \Delta \lambda=35$ to 51 across the full spectra.

With the pixel field of view (PFOV) used, the camera observed beyond the extended emission of the entire galaxies so that the foreground emission could be obtained directly from the maps. The total integration time for each of these observations was $1^{\mathrm{h}} 30^{\mathrm{m}}$.

We reduced the ISOCAM data of NGC 1569, NGC 1140 and II Zw 40 and LMC 30 Dor as well as the already published SMC N 66. SMC B\#1 is taken directly from Reach et al. (2000). For the data treatment we used and adapted the CIR (CAM Interactive Reduction; Version: JAN01; Chanial 2003) package incorporating private IDL routines. Great care was taken to examine the data between each step of the processing in order to verify the data reduction and to look for evidence of artifacts. Table 1 lists the low metallicity sources used in this study along with some source parameters. We proceeded in the following way:

1) Dark current subtraction: the subtraction of the dark currents is performed using a model which predicts the time evolution for each row of the detector (Biviano et al. 1998), taking into account drifts along each orbit and each revolution. The correction implemented in CIR is a second order correction avoiding negative values.

2) Cosmic ray impacts: we masked the glitches using multiresolution median filtering (Starck et al. 1999) on each block of data after slicing the cube. This method works well for common glitches except for faders and dippers (Claret et al. 1998). In addition, we perform manual deglitching after the transient correction (see below), examining the temporal cut for each pixel and masking the bad pixels. Thus, we were able to remove the remaining glitches which were not found by the automatic method. Moreover, we removed the slowly decreasing tails after each glitch. These were not always masked by the algorithm. We also examined the pixels in the vicinity of all of the strong glitches.

3) Transient effects: ISOCAM is subject to systematic memory effects due to the very long time needed for the signal to stabilize. We corrected this using the Fouks-Schubert method (Coulais \& Abergel 2000) which provides good photometric accuracy without any fitting. We noticed that the corners and the edges of the field were not sufficiently illuminated, thus we mask the borders of the detector to allow proper flat-fielding and background subtraction.

4) Flat-fielding: the galaxies were observed with a single pointing. Therefore, we could not use redundancy to build a flat-field for the entire detector. We computed a hybrid flat-field image placing a mask on the source and computing a flat field outside this mask from the median of the temporal cut for each pixel. For the pixels which were on-source, the flat-field response was set to the corresponding calibration flat-field.

5) Flux conversion: the conversion from Analog Digital Units to $\mathrm{mJy} /$ pixel was performed using the standard in-flight calibration data base.
6) Sky subtraction: the sky contributions can, in principle, contain contributions from our Galaxy, background galaxies, and Galactic zodiacal light which is usually the dominating source. To remove the sky contribution, the source was masked and, for a given wavelength, the median of the pixels which are offsource were subtracted from each pixel. A $\sigma_{\text {zodiacal }}$ was determined for the calculation of the total uncertainties (below in paragraph 8), where $\sigma_{\text {zodiacal }}=\sigma_{\text {bkg }} / \sqrt{N_{\text {bkg }}} . N_{b k g}$ is the number of pixels used to measure the sky background and $\sigma_{\mathrm{bkg}}$ is the $1 \sigma$ uncertainty in the background determination.

7) Filtering: we apply a multiresolution filtering method from the MR/1 package (Starck et al. 1998). Each image corresponding to a given wavelength was filtered with a threshold of $3 \sigma$. The final spectra are shown in Figs. 1 and 2.

8) Evaluation of uncertainties: to estimate the uncertainty $\Delta F_{v}(\lambda)$ on the net flux $F_{v}(\lambda)$ integrated in a circular aperture $\Theta$, we quantify the various contributions induced by the data reduction steps and the remaining errors. We propagate the statistical fluctuations for each pixel $(i, j ; \lambda)$ at a given wavelength $\lambda, \sigma_{\text {rms }}(i, j ; \lambda)$ along each step of the processing. The total uncertainty due to these fluctuations on $F_{v}(\lambda)$ in $\Theta$ is $2 \sigma_{\mathrm{rms}}$ or $\Delta F_{v}^{\mathrm{rms}}(\lambda)=2 \times \sqrt{\sum_{(i, j) \in \Theta} \sigma_{\mathrm{rms}}^{2}(i, j ; \lambda)}$. We estimate the error due to the sky subtraction (described above in paragraph 6) using the standard deviation, $\sigma_{\text {zodiacal }}$ of the distribution of the points used to compute the median. This error is $\Delta F_{v}^{\text {zodiacal }}(\lambda)=2 \times \sqrt{\sum_{(i, j) \in \Theta} \sigma_{\text {zodiacal }}^{2}}=\sqrt{N_{\Theta} \sigma_{\text {zodiacal }}^{2}}$ where $N_{\Theta}$ is the number of pixels inside the aperture $\Theta$. The uncertainty due to remaining memory effects was estimated by considering the amplitude of the variations of each pixel up to $3 \sigma_{\text {rms }}, \delta F_{3 \sigma}(i, j ; \lambda)$ within a block of data. We also take into account the time needed for the signal to stabilize using a fac-

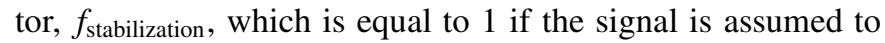
be stabilised and equals $(120 \mathrm{~s}) / \Delta t$ if not stabilised (Roussel et al. 2001a). The uncertainty due to transients is, finally, $\Delta F_{v}^{\text {transients }}(\lambda)=\sqrt{\sum_{(i, j) \in \Theta}\left(\delta F_{3 \sigma}(i, j ; \lambda) \times f_{\text {stabilization }}(\lambda)\right)^{2}}$. The total uncertainty on the net flux is the sum of these different contributions plus $5 \%$ of the flux due to absolute calibration errors and 5\% due to variations along each orbit Siebenmorgen et al. (2000):

$$
\begin{aligned}
\Delta F_{v}(\lambda)= & {\left[\left(\Delta F_{v}^{\mathrm{rms}}(\lambda)\right)^{2}+2 \times\left(0.05 \times F_{v}(\lambda)\right)^{2}\right.} \\
& \left.+\left(\Delta F_{v}^{\mathrm{zodiacal}}(\lambda)\right)^{2}+\left(\Delta F_{v}^{\mathrm{transients}}(\lambda)\right)^{2}\right]^{1 / 2}
\end{aligned}
$$

Table 2 gives an example of the level of the uncertainties for each step, and total, for NGC 1569.

9) Comparison with IRAS $12 \mu \mathrm{m}$ : to verify the photometry of our newly-presented sources, we integrate our CVF specrta over the $12 \mu \mathrm{m}$ IRAS band and measure the flux of the resulting image in the same aperture. For NGC 1569, we find a flux of $(880 \pm 200)$ mJy using our ISOCAM spectra. The IRAS $12 \mu \mathrm{m}$ flux given by Melisse \& Israel (1994) is $508 \mathrm{mJy}$ and is flagged by the authors to be uncertain. The IRAS flux given by Hunter et al. (1989) is $F_{12 \mu \mathrm{m}}=(880 \pm 100)$ mJy which is in excellent agreement with the ISOCAM spectrum. For II Zw 40, we find a flux of $(290 \pm 60)$ mJy integrated in the $12 \mu \mathrm{m}$ IRAS band and the IRAS flux given by Melisse \& Israel (1994) is 

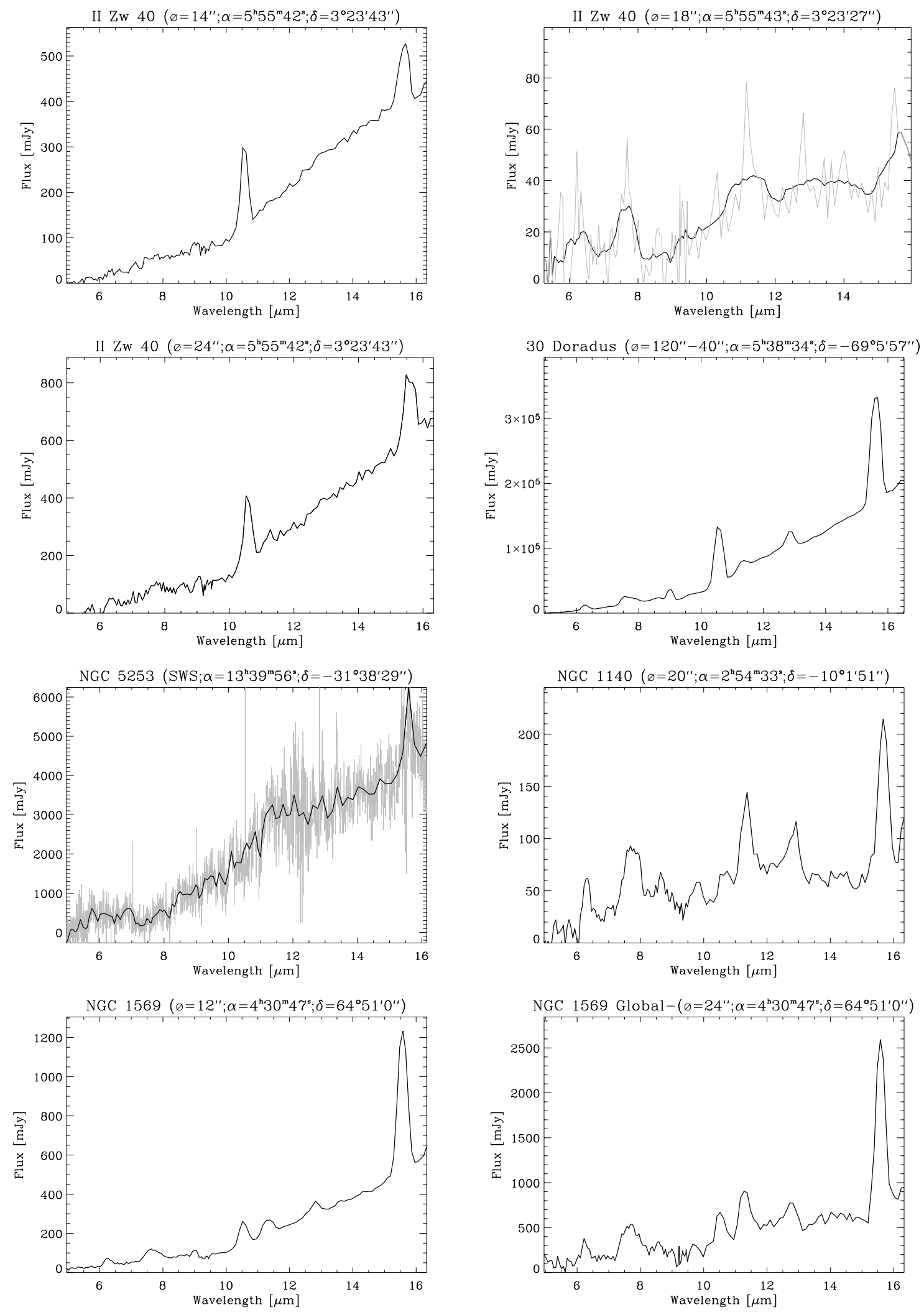

Fig. 1. ISOCAM MIR spectra of dwarf galaxies. The position of the central pointing as well as the diameter of the circular aperture are indicated next to the name of the source, above each spectrum. The spectrum of II $\mathrm{Zw} 40$ in the upper right panel of is taken toward the merger tail. Both the smoothed (black) and original (grey) profiles are displayed (see Sect. 3.3.3 and Fig. 7). Two profiles are displayed for NGC 5253: the original SWS spectrum is the grey profile (Crowther et al. 1999) and the black profile is the original spectrum smoothed to the spectral resolution of ISOCAM. The spectrum of the peak 12" region of NGC 1569 (lower left) is shown in contrast with the integrated emission of the "extended" region of NGC 1569 (lower right): the full galaxy with the central 24" removed. 

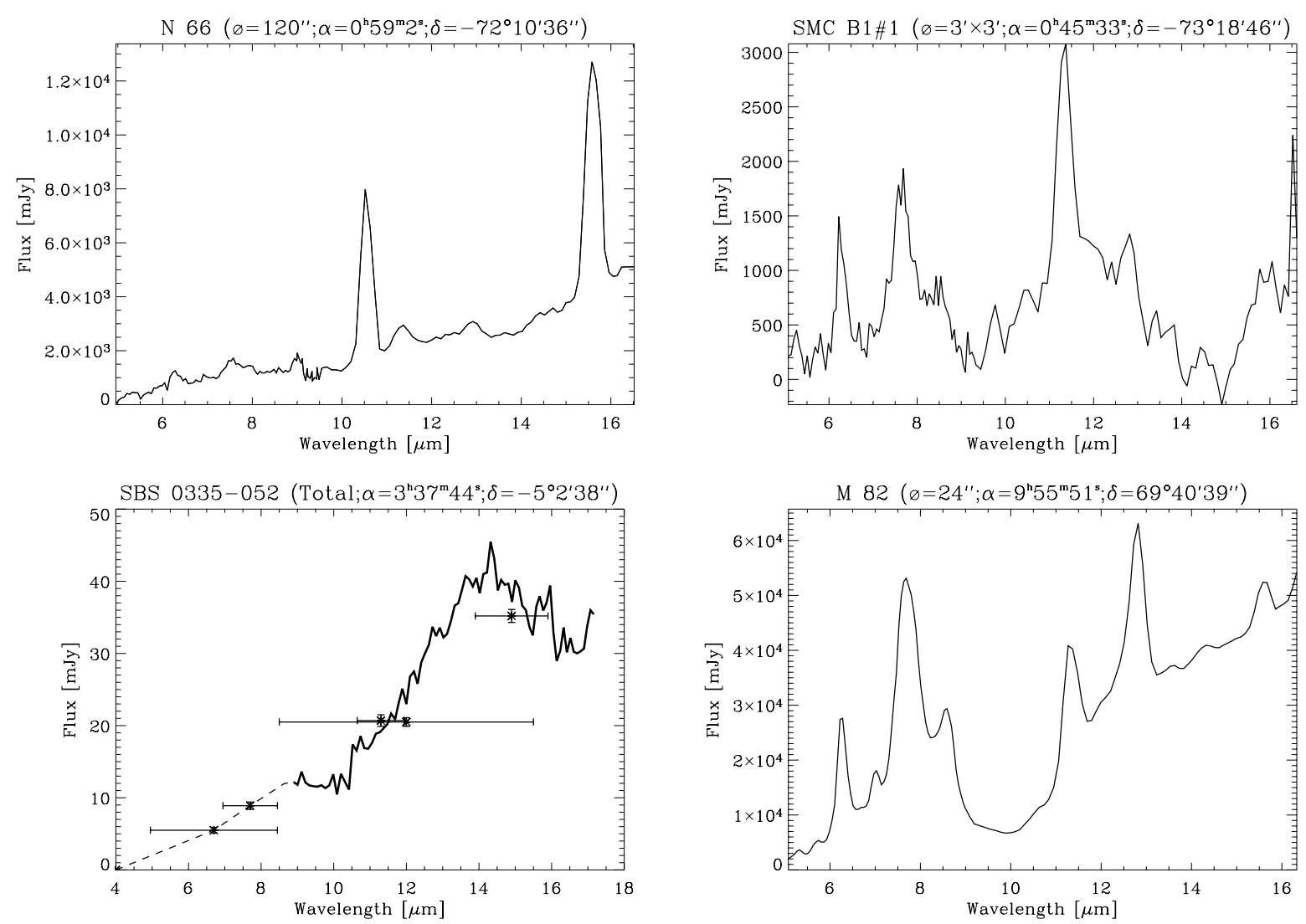

Fig. 2. ISOCAM MIR spectra from the SMC: SMC N 66 and SMC B\#1 and the very low metallicity galaxy, SBS 0335-052 (Thuan et al. 1999). For comparison with a metal rich starburst galaxy, we also include the global ISOCAM CVF spectra of M 82 . The position of the central pointing as well as the diameter of the circular aperture are indicated next to the name of the source, above each spectrum. For comparison with a metal rich starburst galaxy, we also include the ISOCAM CVF spectra of the central region of M 82 in Fig. 2. The spectral features identified in the spectra are discussed in Sect. 3.2.

Table 2. Example of the various uncertainties quantified for the global CVF of NGC 1569.

\begin{tabular}{lllll}
\hline \hline & rms & Transients & Zodiacal & Total \\
\hline Average & $13 \%$ & $22 \%$ & $3 \%$ & $27 \%$ \\
$\lambda=16 \mu \mathrm{m}$ & $13 \%$ & $9 \%$ & $2 \%$ & $17 \%$ \\
$\lambda=4 \mu \mathrm{m}$ & $66 \%$ & $31 \%$ & $13 \%$ & $75 \%$ \\
\hline
\end{tabular}

$F_{12 \mu \mathrm{m}}=415 \mathrm{mJy}$. This is higher than our measured flux value and may indicate a substantial extended very low level $12 \mu \mathrm{m}$ flux. Beck et al. (2002) observed this galaxy with the Keck 1 Telescope and found a total flux of $F_{11.7 \mu \mathrm{m}}=240 \mathrm{mJy}$. The ground-based narrow band measurements are consistent with our $11.7 \mu \mathrm{m}$ flux from the ISOCAM CVF spectrum. For NGC 1140, we find a flux of $(96 \pm 30) \mathrm{mJy}$, in agreement with the $12 \mu$ m IRAS flux value from Melisse \& Israel (1994), who measure $F_{12 \mu \mathrm{m}}=73 \mathrm{mJy}$.

\section{Spectral analysis}

The MIR wavelength range covered by ISOCAM CVF ranges from $\lambda=5$ to $16 \mu \mathrm{m}$ and contains a rich assortment of tracers of various components of gas and dust in galaxies. Fine structure emission lines arising from H II regions, silicate features, aromatic hydrocarbon features, dust continuum and possibly stellar continuum, are all interwoven into a MIR spectrum which can be disentangled to extract clues of the physics of star formation. When observing galaxies, the telescope beam will contain a variety of components of the ISM and star formation regions. The relative contributions of each component depends on many physical parameters, including metallicity, star formation activity and ISM structure - all conspiring together to govern the global observed emission.

Two components of dust emission can be present in MIR spectra:

- The aromatic MIR band features, the major ones being at $\lambda=6.2,7.7,8.6,11.3,12.6 \mu \mathrm{m}$, are thought to come from large molecules or very small grains of carbonaceous origins, for example, PAHs (Léger \& Puget 1984; Allamandola et al. 1985). Due to their very small nature, these particles undergo thermal fluctuations of a few hundreds of degrees $\mathrm{K}$ upon single photon absorption (e.g. Sellgren et al. 1990; Verstraete et al. 2001). From spectroimaging of Galactic regions, the PAHs are seen to peak in photodissociation regions (PDRs) - the interface envelopes between H II regions and molecular clouds (Cesarsky et al. 1996b). 
- Very Small Grains (Désert et al. 1990,VSGs;), wavelengths longward of $\lambda \simeq 10 \mu \mathrm{m}$. The VSGs can be either in thermal equilibrium with the radiation field, or stochastically heated to high temperatures, depending on the radiation field and the sizes of the grains. In the Galaxy, these particles range in size from $\lambda=1 \mathrm{~nm}$ to $10 \mathrm{~nm}$ and are thought to be mostly stochastically heated in the diffuse ISM (Désert et al. 1990). This component is seen to peak in H II regions, when observed with sufficient spatial resolution (Cesarsky et al. 1996b; Verstraete et al. 2001).

Both PAHs and VSGs are heated by UV photons in the vicinity of active star formation regions, with the PAHs observed to peak in the PDRs, and the VSGs emitting prominently in the nebular regions (Cesarsky et al. 1996b; Verstraete et al. 1996). PAHs can also be excited by optical photons, as shown to be the case in reflection nebulae (Uchida et al. 1998), and by their presence in elliptical galaxies (e.g. Athey et al. 2002; Xilouris et al. 2004).

In the disks of spiral gaxies, the PAH emission bands are a very prominent component of the ISM, while the continuum due to the VSGs is less so (e.g. Dale et al. 2000; Roussel et al. 2001b; Vogler et al. 2005). In the nuclei of spiral galaxies, starbursts or active galactic nuclei, various nebular fine structure lines can also be observed in the ISO MIR wavelength range, notably the $[\mathrm{Ne} \mathrm{II}] \lambda 12.81 \mu \mathrm{m},[\mathrm{Ne} \mathrm{III}] \lambda 15.56 \mu \mathrm{m}$, [Ar III] $\lambda 8.99 \mu \mathrm{m},[$ Ar II] $\lambda 6.98 \mu \mathrm{m}$ and [S IV] $\lambda 10.51 \mu \mathrm{m}$ lines (Genzel et al. 1998; Thornley et al. 2000; Laurent et al. 2000; Rigopoulou et al. 2002; Sturm et al. 2002; Lutz et al. 2003; Förster Schreiber et al. 2003; Vogler et al. 2005).

\subsection{Comparison with a Galactic HII region/PDR: $M 17$}

Lacking sufficient spatial resolution to isolate various physical components within galaxies, it is also possible to decompose a global spectra with known template spectra representative of, for example, H II regions, PDR regions, diffuse regions, etc. We use ISOCAM spectra from the Galactic region, M 17 SW, as one way to interpret the spectra of the dwarf galaxies, NGC 1569, NGC 1140 and II Zw 40, which exhibit very different MIR properties.

M 17 has been studied in great detail (Cesarsky et al. 1996b): it contains an H II region and PDR interface adjacent to a molecular cloud, illuminated by an $\mathrm{O} 3$ star and is viewed almost edge-on. With the spatial resolution of ISOCAM, individual physical regions can be isolated and used as templates to interpret the dwarf galaxy spectra. ISOCAM spectra of a typical H II region and a PDR region, extracted from the M 17 ISOCAM image (Cesarsky et al. 1996b) are shown in Fig. 3. $\mathrm{PAH}$ emission peaks in the PDR interfacing the $\mathrm{H}$ II region and molecular cloud, also extending somewhat into both regions. Inside the $\mathrm{H}$ II region, the continuum emission from the hot VSGs is the principal dust component while the PAH emission is supressed, presumably destroyed by the hard, intense radiation field within H II regions (e.g. Leach 1987; Voit 1992; Siebenmorgen et al. 2004). The template spectra were normalised by the total energy in the wavelength range and best combinations of these characteristic template spectra were then determined, using a least squares fit to infer the proportion of ionised and PDR material in the MIR spectra. When observing galaxies, an ensemble of physical components are in the beam, making it difficult to isolate the physical processes.

The global spectrum of II Zw 40 is clearly dominated by contributions from H II regions as evidenced by the steeplyrising continuum and the prominent [Ne III] and [S IV] lines. Our crude model finds the MIR emission of II $\mathrm{Zw} 40$ is made up of emission from a factor of 30 more characteristic $\mathrm{H}$ II regions compared to PDR-type material when matching the shape of the continuum and the PAH bands on the global scale (Fig. 3). Modeling the spectrum toward the central peak only, the ratio of $\mathrm{H}$ II regions to PDRs in the beam reduces to 20, since in this case, we are focusing on most of the PDR gas as well as the ionised gas, all peaking toward the unresolved center. There appears to be a non-negligible component of $e x-$ tended ionised gas throughout the galaxy, when comparing the global and peaked spectra. Only traces of PDRs can be observed in the spectra in the form of very low level PAH bands in II Zw 40.

The global MIR spectrum of NGC 1569 is also dominated by $\mathrm{H}$ II regions, with $20 \%$ PDR origin and $80 \% \mathrm{H}$ II region origin when matching the PAH emission and the VSG continuum in the global spectrum (Fig. 3). Note that when the peak spectrum is modeled, the contribution from $\mathrm{H}$ II regions increases to a factor of 10 above that of PDRs. The [Ne III] and [S IV] lines are particularly intense in the dwarf galaxies, and are not reproduced well by simply using the template spectrum of the nebular emission surrounding the single O3 star from M 17. While the overall shape of the II Zw 40, NGC 1569 and M 17 $\mathrm{H}$ II region spectra are similar, the differences in metallicity can explain the effects of the ionic lines. For example, simply multiplying up the $\mathrm{H}$ II region template spectrum of M 17 to match those of the continuum and ionic lines of the dwarf galaxies, is not sufficient. The ionic lines require further modeling to be able to draw any quantitative information here. The nebular line characteristics are discussed further in Sect. 4.2.

The MIR spectrum of NGC 1140, which has the most prominent PAH emission and obviously flatter VSG continuum, is composed of equal contributions of a PDR component and $\mathrm{H}$ II regions (Fig. 3). Since the extended emission is not very well resolved here, there is little difference in the MIR characteristic when inspecting the global spectrum or the peak spectrum.

\subsection{MIR component separation}

To analyze these spectra and separate the various MIR components, we followed the fitting procedure detailed in Galliano (2004) and Galliano et al. (2005). We proceeded as follows:

1. Small grain continuum: VSGs A modified black body is used to model the very small grains (VSGs). We use an emissivity, $\beta=1$, assuming that these very small grains are mainly carbonaceous. The fit is not very sensitive to the value of $\beta$. We have also tried a solution with a power-law instead of a black body to model the very small grain continuum, however, this solution is mathematically less stable 

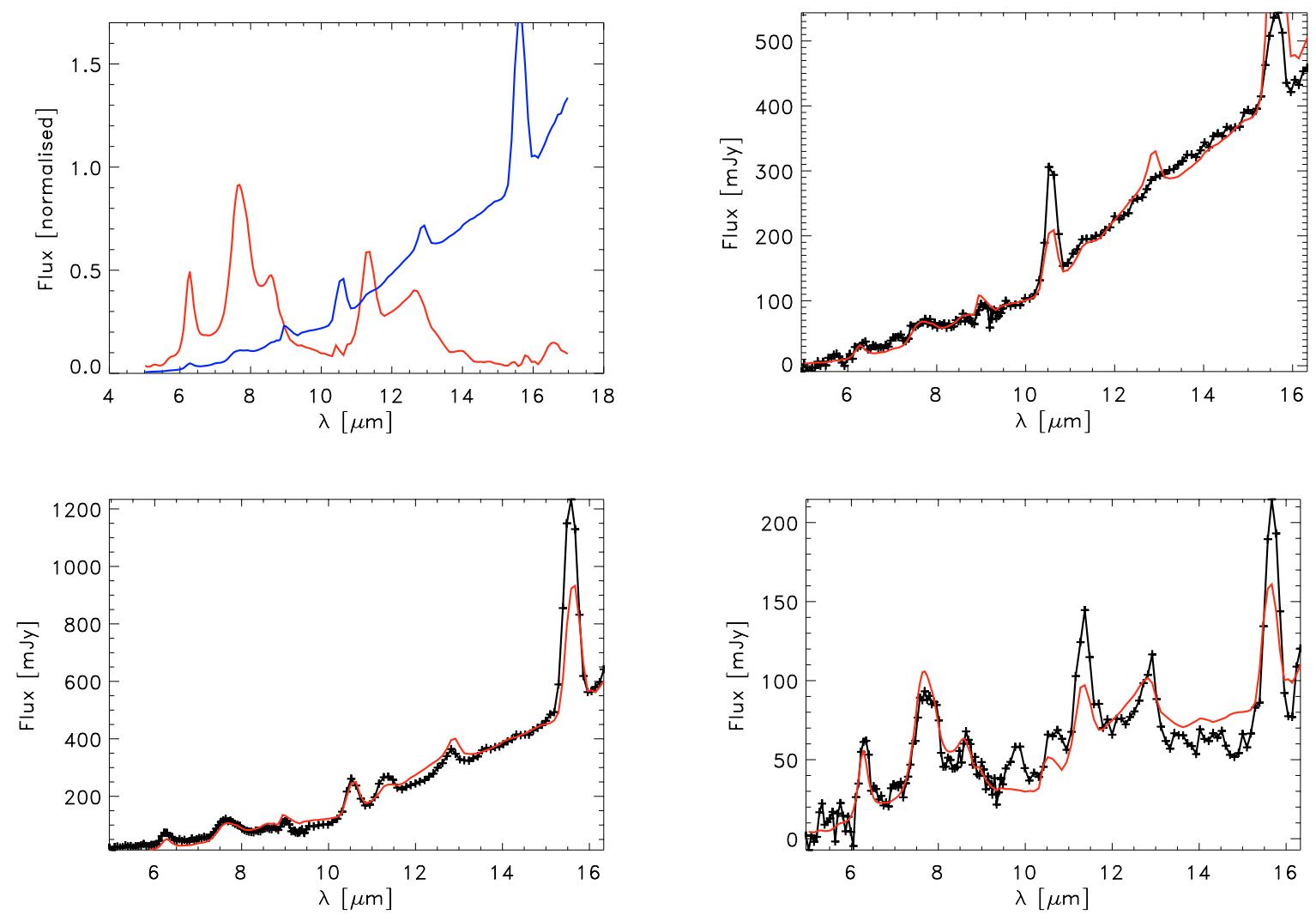

Fig. 3. Template H II region (blue; steeply-rising continuum) and PDR spectra (red; flat continuum) from M 17 (upper left), used as examples to interprete the global MIR spectra of 3 dwarf galaxies in our sample: II Zw 40 (top right), NGC 1569 (lower left) and NGC 1140 (lower right). The galaxy spectra are global averages with the observations represented by black profiles and crosses and the red profiles are the total spectra modeled with combinations of the template H II region and PDR spectra. The H II template was extracted in a $30^{\prime \prime} \times 30^{\prime \prime}$ aperture centered on $\left(18^{\mathrm{h}} 20^{\mathrm{m}} 26^{\mathrm{s}} 2,-16^{\circ} 11^{\prime} 25.4^{\prime \prime}\right)$, and the PDR template, in a $30^{\prime \prime} \times 30^{\prime \prime}$ aperture centered on $\left(18^{\mathrm{h}} 20^{\mathrm{m}} 19^{\mathrm{s}} 9,-16^{\circ} 12^{\prime} 17.5^{\prime \prime}\right)$. See the electronic version of the Journal for color version.

for noisy spectra. Throughout the paper, where we refer to the VSGs in images or plots, we are referring to this fitted VSG component, integrated between 10 and $16 \mu \mathrm{m}$.

2. Nebular lines: Gaussian functions are applied to the fitting of the ionic lines. We fixed the central wavelength and computed the line widths and the line intensites. The lines included for the dwarf galaxies are: [Ne III] $] 15.56 \mu \mathrm{m}$, $[\mathrm{Ne}$ II $] \lambda 12.81 \mu \mathrm{m},[\mathrm{S} \mathrm{IV}] \lambda 10.51 \mu \mathrm{m},[\mathrm{Ar}$ III] $] \lambda .99 \mu \mathrm{m}$ and [Ar II] $\lambda 6.98 \mu \mathrm{m}$. At the spectral resolution of ISOCAM, the [Ne II] $\lambda 12.81 \mu \mathrm{m}$ is blended with the $12.6 \mu \mathrm{m}$ PAH band. The fitted width of the line with the best signal-to-noise, usually the [Ne III] or [S IV] line, is presumed to be the fixed width of the other gas lines.

3. PAH bands: PAH bands are fitted with Lorentzian functions by fixing the central wavelengths and leaving the width and intensity as free parameters. The bands taken into account here are: $5.3 \mu \mathrm{m}, 5.7 \mu \mathrm{m}, 6.2 \mu \mathrm{m}, 7.7 \mu \mathrm{m}, 8.6 \mu \mathrm{m}, 11.3 \mu \mathrm{m}$, $12.0 \mu \mathrm{m}, 12.6 \mu \mathrm{m}, 13.6 \mu \mathrm{m}$ and $14.3 \mu \mathrm{m}$. Note that where we refer to PAH bands in the following images or plots, we are referring to the integrated intensity of this whole fitted component - essentially all of the PAH bands.

4. The sum of these components is reddened with a mid-IR extinction curve of Mathis (1990), with the silicate profiles of Dudley \& Wynn-Williams (1997). This accounts for the intrinsic extinction of the galaxies. The magnitude of the extinction is a free parameter.

This fit is done after redshift correction, where necessary. Each component is fitted simultaneously to obtain the best $\chi^{2}$. Examples of the line fits and their residuals are shown in Fig. 4.

The residuals, shown in the small panels in Fig. 4, appear relatively uniform, except for a noisy potential feature near $10 \mu \mathrm{m}$ coinciding in wavelength with the $\mathrm{SiO}$ stretching silicate band, which is not included in the model for the emission spectra. It also appears close in wavelength to the position where the 2 grating spectrometers of the ISOCAM overlap (one CVF: $\lambda=5 \mu \mathrm{m}$ to $9.5 \mu \mathrm{m}$ and the other CVF: $\lambda=9 \mu \mathrm{m}$ to $16 \mu \mathrm{m}$ ). The residual feature near $10.5 \mu \mathrm{m}$ seen II $\mathrm{Zw} 40$ (Fig. 4), is from the fitted [S IV] line.

The ionic lines are very prominent in the spectra of dwarf galaxies (Fig. 4; Table 3), while, for the most part, there is a paucity of PAHs, on size scales traced by the ISOCAM beam as well as on global scales. NGC 1140 seems to be somewhat of an exception (Fig. 1), as the PAHs are rather conspicuous. Normally, the PAH bands figure prominently in the spectra of dust-rich starburst and spiral galaxies (e.g. Laurent et al. 2000; Sturm et al. 2000; Förster Schreiber et al. 2003). While NGC 1569 shows low level PAH emission with respect 

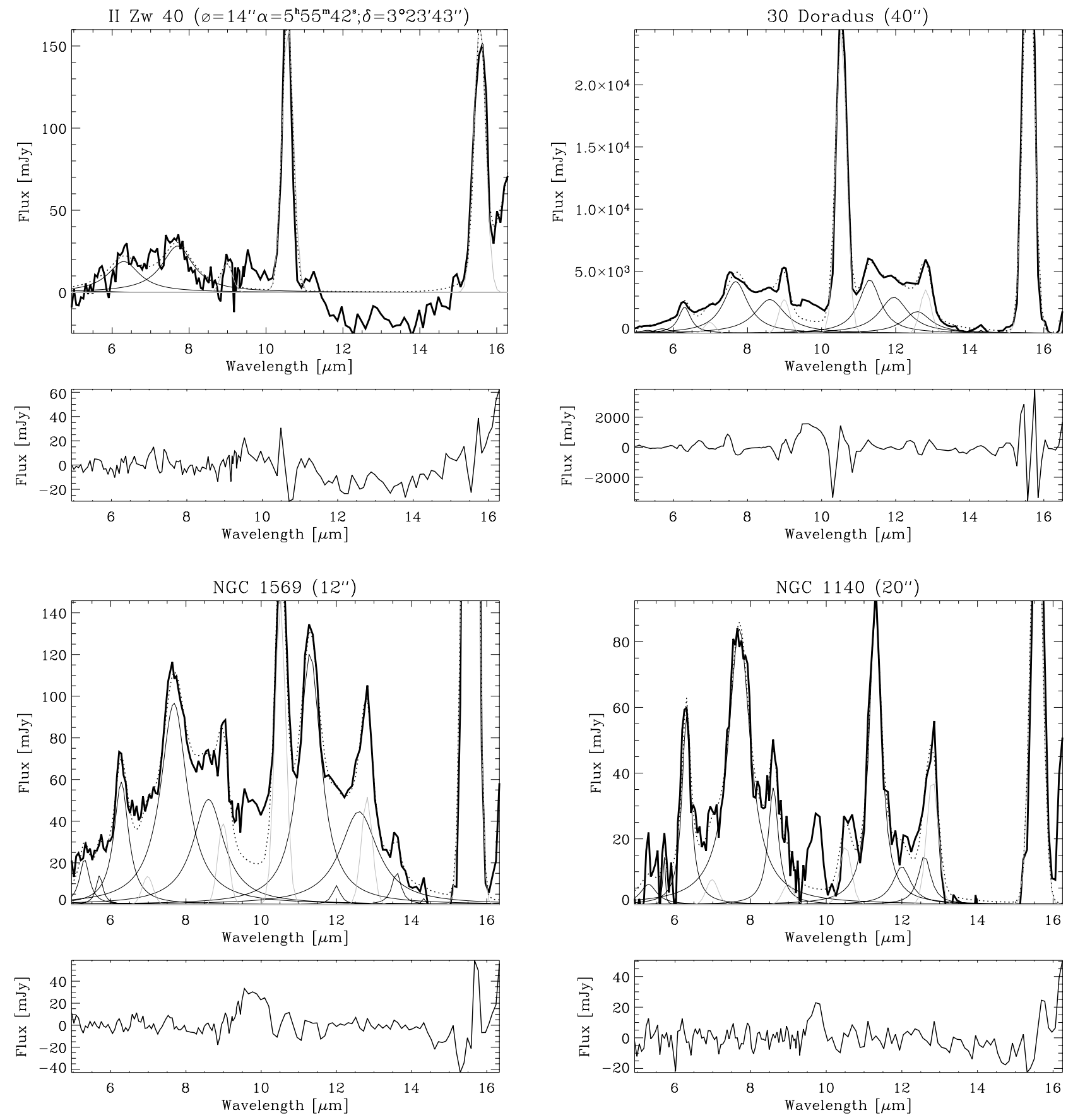

Fig. 4. Examples of the fits of the ionic lines and the PAH bands. The thick black line is the original spectrum after subtraction of the continuum (see Sect. 3.2 for line and continuum fitting procedure); thin black lines are the Lorentzian PAH band fits; thin grey lines are the Gaussian ionic line fits. The sum of the model components are the dotted lines. The residuals after the line and continuum fitting procedures are also shown in the smaller panels beneath the complete spectra.

to the rising continuum, toward the central region (lower left of Fig. 1), the extended emission of NGC 1569, shown toward the lower right of Fig. 1, demonstrates that PAHs are rather conspicuous, while the continuum is flatter. Globally, NGC 1569 resembles the spectrum toward the central peak. The contrast between the MIR spectra of the low-metallicity starbursts and the nearby metal-rich starburst galaxy, M 82, for example, can be observed in Fig. 2. The MIR spectrum toward the central $24^{\prime \prime}$ of M 82 is clearly dominated by material originating from the disk, which shows dominant PAH emission (e.g. Laurent et al. 2000; Roussel et al. 2001b; Dale \& Helou 2002; Förster Schreiber et al. 2003; Vogler et al. 2005). On a global scale, the M 82 MIR spectra also looks like the central 24" with similarly prominant PAH bands, but with somewhat lower rising continuum longward of $14 \mu \mathrm{m}$. When viewed on global scales, the dwarf galaxies are not dominated by PAHs, but by the VSG continua characteristic of H II regions (e.g. Peeters et al. 2002; Martín-Hernández et al. 2002a). 
Table 3. Ionic line intensities in $10^{-15} \mathrm{~W} \mathrm{~m}^{-2}$ for the low metallicity galaxies (global values). In the case of $30 \mathrm{Dor}$ and SMC N 66, the fluxes are integrated over a $120^{\prime \prime}$ circular aperture. These line intensities are corrected for the internal extinction. This extinction correction is negligible in all the sources, except II $\mathrm{Zw} 40$, and M 82. The upper limits are $3 \sigma$ values.

\begin{tabular}{lllll}
\hline \hline & {$[\mathrm{Ne}$ III $]$} & {$[\mathrm{Ne}$ II $]$} & {$[\mathrm{S} \mathrm{IV}]$} & {$[$ Ar III] } \\
\hline NGC 1140 & $0.8 \pm 0.13$ & $0.36 \pm 0.55$ & $0.19 \pm 0.05$ & $\lesssim 8.2 \times 10^{-3}$ \\
NGC 1569 & $14.7 \pm 1.0$ & $1.90 \pm 0.28$ & $6.7 \pm 1.2$ & $1.4 \pm 0.6$ \\
NGC 5253 & $8.7 \pm 0.6$ & $\lesssim 0.7$ & $\lesssim 2.2$ & $\lesssim 0.4$ \\
II Zw 40 & $1.48 \pm 0.12$ & $\lesssim 0.26$ & $5.24 \pm 0.53$ & $\lesssim 2.2$ \\
30 Dor & $(1.04 \pm 0.04) \times 10^{3}$ & $(1.87 \pm 0.07) \times 10^{2}$ & $(1.35 \pm 0.05) \times 10^{3}$ & $(2.56 \pm 0.12) \times 10^{2}$ \\
SMC N 66 & $42.0 \pm 2.4$ & $\lesssim 3.5$ & $66.5 \pm 4.1$ & $15.0 \pm 2.9$ \\
SMC B\#1 & $\lesssim 3.6$ & $6.8 \pm 1.0$ & $6.0 \pm 0.9$ & $\lesssim 2.3$ \\
M 82 & $54.0 \pm 2.2$ & $368 \pm 14$ & $58.9 \pm 3.2$ & $\lesssim 24$ \\
\hline
\end{tabular}

In resolved regions of the closest low-metallicity galaxies, the SMC and the LMC, PAHs are indeed present at low levels: SMC B\#1 region (Reach et al. 2000, and our Fig. 2) and SMC N 66 (Contursi et al. 2000, and our Fig. 2) and 30 Dor in the LMC (Fig. 1) and other regions in the LMC (Vermeij et al. 2002). In the Local Group dwarf galaxy, NGC 1569, PAH band emission is likewise evident, yet relatively supressed. It may not be surprising to see more conspicuous local PAH band emission from the PDRs illuminated by H II regions in low metallicity regions at very high spatial resolution.

The prominent ionic lines and significant continua observed on large scales in these galaxies compel us to compare the observed MIR spectra with those of Galactic H II regions. The presence of PAHs in some galaxies also implies some contribution from the molecular clouds/PDRs in the vicinity. In order to have a better idea as to which ISM components are traced by the MIR spectra in the beam, we compare some of these spectra with the Galactic region, M 17 where the H II region and PDR region are well resolved with ISOCAM (Cesarsky et al. 1996b).

\subsection{Spatial distribution of various MIR components}

For some of the nearby, well-resolved sources, such as NGC 1569, II Zw 40 and 30 Dor, we study the spatial distribution of the various physical components in more detail. There was not sufficient signal to deconvolve each individual image of the CVF cubes, so the spatial resolution of the images were degraded, as specified below. The MIR spectrum of each pixel was then computed (as described in Sect. 3.2) and the spectral analysis was performed to extract the various physical components for each pixel. The maps of the PAHs or ionic lines consist of the integrated intensities of those complete components. The VSG maps are constructed from the extracted VSG continuum component, integrated from 10 to $16 \mu \mathrm{m}$, to characterise the slope of the VSG continuum. To generate the ratio maps, we computed the ratios of components only for the pixels where the flux of the denominator was greater than $3 \%$ of the maximum to avoid non relevant values at the edge of the maps due to low flux values.

Inspection of the individual spectra shows that considering the low signals at short wavelengths and the uncertainty values, there is negligible local contribution at the shortest wavelengths, $5 \mu \mathrm{m}$, from any possible cooler, evolved stellar population. The physical components which we are displaying as images here, are all emitting at wavelengths $>6 \mu \mathrm{m}$. Thus, if our assumption concerning negligible local stellar contribution at short wavelengths was incorrect, these maps would not be effected.

\subsubsection{NGC 1569}

The study of the physical components in the nearby galaxy, NGC 1569, provides a unique view of the physical components within a starburst dwarf galaxy in some detail. The resolution of our maps was degraded to $10^{\prime \prime}(\sim 100 \mathrm{pc})$, which is roughly equivalent to the beam size at $16 \mu \mathrm{m}$. Final maps for each of these components are shown in (Fig. 5).

At first glance, with the ISOCAM spatial resolution, the ionic, dust and PAH components all peak toward the massive clusters of HII regions to the west (Waller 1991) and follow the starforming ridge, with a secondary peak toward the east (Fig. 5). Super star clusters reside within the peak PAH emission zone, but only much higher resolution data would isolate the precise nature of the MIR component peaks. A variety of ISM components would be sampled toward NGC 1569 within a beam size of $10^{\prime \prime}$, which is $106 \mathrm{pc}$. While the MIR peaks are all located toward the same locations, the extent of the emission and the rate of fall-off varies from component to component. The PAHs have a relatively large extended component, with the low level emission ( $5 \%$ of peak value) ranging out to $80^{\prime \prime}$ to $40^{\prime \prime}$. The VSGs, normally confined to within H II regions, appear relatively more compact. The $[\mathrm{Ne}$ III $] \lambda 15.56 \mu \mathrm{m}$ and the [Ne II] $\lambda 12.81 \mu \mathrm{m}$ line emission, however, is very extended - on the same scale as the PAH emission. There is a substantial pervasive, diffuse ionised medium throughout the galaxy. Notice the western filament extensions seen in the [Ne II] and PAH images. These are spatially coincident with the superwinds impresively exhibited in the $\mathrm{H} \alpha$ image of Waller (1991).

Maps of the ratios of these components can shed some light on the behavior of the MIR tracers (Fig. 5). The $[\mathrm{Ne} \mathrm{III}] /[\mathrm{Ne} \mathrm{II}]$ ratio looks relatively flat along the star formation ridge. As this ratio is related to the hardness of the ISRF (see Sect. 4.2), the hard ISRF is not very well confined to only 

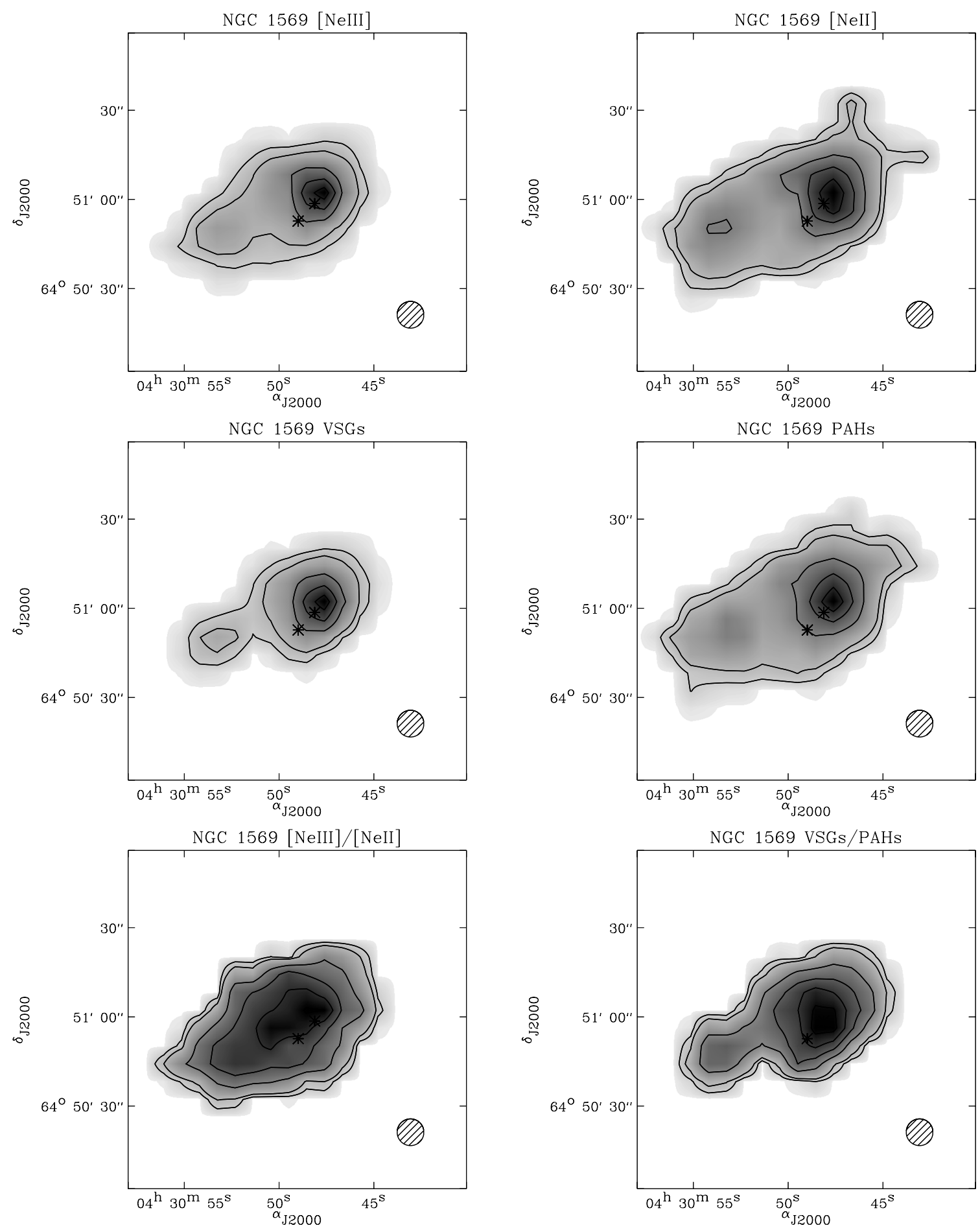

Fig. 5. Maps of some physical components for NGC 1569. Up-left: [Ne III]; up-right: [Ne II]; middle-left: VSG continuum; middle-right: PAHs; lower-left: [Ne III]/[Ne II]; lower-right: VSGs/PAHs; The contour levels are 5, 10, 30, 50, 70 and $90 \%$ of the peak intensity. The peak intensities are: $[\mathrm{Ne}$ III $]=2.2 \times 10^{-15} \mathrm{~W} \mathrm{~m}^{-2}$ beam $^{-1},[\mathrm{Ne} \mathrm{II}]=2.3 \times 10^{-16} \mathrm{~W} \mathrm{~m}^{-2}$ beam $^{-1}, I(\mathrm{VSG})=5.2 \times 10^{-14} \mathrm{~W} \mathrm{~m}^{-2} \mathrm{beam}^{-1}, I(\mathrm{PAH})=$ $1.4 \times 10^{-14} \mathrm{~W} \mathrm{~m}^{-2}$ beam $^{-1},[\mathrm{Ne} \mathrm{III}] /[\mathrm{Ne} \mathrm{II}]=9.6, I(\mathrm{VSG}) / I(\mathrm{PAH})=3.6$. The resolution has been degraded to the beam size at $16 \mu \mathrm{m}$ which is roughly $10^{\prime \prime}(100 \mathrm{pc})$. The asterisks are the position of the super star clusters SSC-A and SSC-B.

the most compact sources, but is more extended. This effect can be noted in the image of the VSGs/PAHs. The central region of the galaxy has a high ratio of VSGs/PAHs, i.e., relatively lower level of PAHs in the central, most active area, where the VSG hot grain continuum is also elevated. The photons responsible for the high level VSG continuum observed in the low metallicity galaxies would also excite the MIR PAH modes, if the PAHs are sufficiently abundant. Further from the star formation sites, the PAH component begins to dominate over the VSGs. This effect could also be interpreted in light of PAH 
destruction due to the hardness of the radiation field. This possibility is discussed further in Sect. 4.3. The VSG distribution seems to be more sharply peaked with a smaller FWHM than that of the [Ne III] line (Fig. 5).

\subsubsection{Dor}

The images of the MIR components of 30 Dor were constructed in the manner described above for NGC 1569. The angular scale of $10^{\prime \prime}$ corresponds to a linear size of $2.4 \mathrm{pc}$. This angual scale should allow us to study the details in the region around the massive star cluster R136 and the surrounding PDR/molecular cloud region (Fig. 6). The region directly toward the R136 star formation, has a minimum in emission in all of the tracers, illustrating the effects of the massive star formation activity with winds efficiently blowing out the ISM in the immediate surroundings (Fig. 6). The distribution of the [Ne III] and that of the [Ne II] emission differ close to the R136 cluster, where the [Ne III] emission is more prominent. The difference in excitation potentials of the [Ne III] and [Ne II] lines, is illustrated nicely by the partial shell of $[\mathrm{Ne}$ II] emission around the cluster, which has a peak displaced further from the exciting cluster than that of the [Ne III]. The PAHs have peaks beyond the peak of the VSG emission. They have a relatively broad distribution which appears to be flatter than that of the VSGs. The hardest form of the radiation field, traced by the $[\mathrm{Ne} \mathrm{III}] /[\mathrm{Ne} \mathrm{II}]$ ratio, is confined to a relatively limited region, about $10 \mathrm{pc}$ from the R136 exciting source. The peaks of the PAH emission avoid the peaks of the $[\mathrm{Ne}$ III]/[Ne II] ratio. This can also be seen in the distribution of the VSGs/PAH, which shows a peak coincident with the peak of the $[\mathrm{Ne}$ III] $] /[\mathrm{Ne}$ II] ratio.

The distribution of the various tracers of the ISM appear to overlap spatially. Even at this spatial resolution, there is not a very distinct separation between the various ISM components at such spatial scales. A very clumpy ISM at smaller spatial scales could be one reason for this. High resolution, groundbased MIR observations could reveal the geometry more accurately.

\subsubsection{Zw 40}

To visualise structure of the MIR emission of II $\mathrm{Zw} 40$, the CVF was integrated to simulate the LW2 broad band (5 to $8.5 \mu \mathrm{m})$ to create a map optimised in signal-to-noise. The overlaping tails of the interacting galaxies could then be readily identified using the R band image of Gil de Paz et al. (2003). The smoothed CVF spectrum shown in Fig. 1 integrated in the overlapping tail region, has a $\mathrm{S} / \mathrm{N}$ of 3.4 at $7.7 \mu \mathrm{m}$ and indicates that the presence of PAHs is more apparent in the overlap region, where the continuum is not very striking, than toward the central MIR peak of the galaxy (Fig. 7). The contrast between the spectra in these 2 regions is seen in Fig. 1 (upper left). The spectrum integrated within a $14^{\prime \prime}$ aperture toward the central MIR peak of the galaxy, shows little evidence for PAH emission, and very strong MIR continuum, and is dominated by emission from the starburst activity. The spectra of the merging tails of the interacting galaxies, on the other hand, shown in Fig. 1 (upper right), contains more ISM material, perhaps indicative of the original disk material of the 2 merging galaxies. Still, globally, the MIR emission is dominated by the ionic lines and hot dust continuum.

\subsection{Contributions of various components in ISOCAM broad bands}

The most popular broad bands that were used to image galaxies with ISOCAM were the $6.7 \mu \mathrm{m}$ LW2 (5.0 to $8.5 \mu \mathrm{m})$ and the $15 \mu \mathrm{m} \mathrm{LW} 3$ (12 to $18 \mu \mathrm{m}$ ) bands. Often it was assumed that the $6.7 \mu \mathrm{m}$ band traces the PAHs, emitting at 6.3, 7.7 and $8.6 \mu \mathrm{m}$, and that the $15 \mu \mathrm{m}$ band traces the VSG continuum. Large extragalactic studies were carried out using these 2 bands (e.g. Laurent et al. 2000; Roussel et al. 2001a; Dale et al. 2000). Here we quantify the contribution of the different components in some useful ISOCAM broad bands, integrating the spectra over the appropriate wavelength ranges, and convolve them with the corresponding broad band filters. This exercise can serve as a useful aide in the interpretation of broad band data from low metallicity regions when MIR spectra are not available.

To evaluate the contribution for any component, integrated intensities of the features and continua were computed, following their decomposition as describled in Sect. 3.2. Table 4 lists the percentage of the flux of the various MIR components, PAH bands, ionic lines and the VSG continua, which are contained within the ISOCAM broad bands LW2 (centered at $6.7 \mu \mathrm{m}$, from 5 to $8.5 \mu \mathrm{m}$ ), LW3 (centered at $15 \mu \mathrm{m}$, from 12 to $18 \mu \mathrm{m}$ ) and the $12 \mu \mathrm{m} \mathrm{LW} 10$ band which resembles the IRAS band and is centered at $12 \mu \mathrm{m}$, from 8 to $15 \mu \mathrm{m}$ ), for the 7 low metallicity sources along with M 82 for comparison. The LW2 band is dominated by the PAH band emission in all but 2 of the galaxies: the global spectra of the more active galaxies, NGC 5253 and II Zw 40 have about $1 / 2$ of their total $6.7 \mu \mathrm{m}$ (LW2) band flux originating from the wings of the Lorentzians of the fitted PAHs, while 1/2 arises from the small grain continua.

However, most of the $15 \mu \mathrm{m}$ (LW3) band flux originates from the hot VSG continua, except for the case of SMC B\#1, which traces the diffuse ISM, thus accounting for the significant contribution from PAHs (e.g. Mattila et al. 1996). Even in NGC 1140, where the continuum is relatively flat (see Fig. 1) the VSGs still make up $75 \%$ of the total LW3 broad band flux. In the active starburst galaxies, NGC 5253 and II Zw 40, as well as the starforming reigons, SMC N 66 and 30 Dor, $85 \%$ to $97 \%$ of the $15 \mu \mathrm{m}$ LW3 flux is originating from the VSG component in the $12 \mu \mathrm{m}$ broad band.

In the $15 \mu \mathrm{m}$ LW3 broad band, the [Ne III] line alone can contribute between $6 \%$ to $10 \%$ of the total flux for the starbursting dwarf galaxies/H II regions, such as NGC 1569, NGC 1140, 30 Dor and SMC B\#1. The [Ne III] $\lambda 15.56 \mu \mathrm{m}$ line is one of the brightest ionic lines in the MIR spectra, along with the [S IV] $\lambda 10.51 \mu \mathrm{m}$ line (see Table 3 ).

The broader, IRAS-type $12 \mu \mathrm{m}$ band, LW10, has at least $37 \%$ of its flux originating from VSGs in all sources 

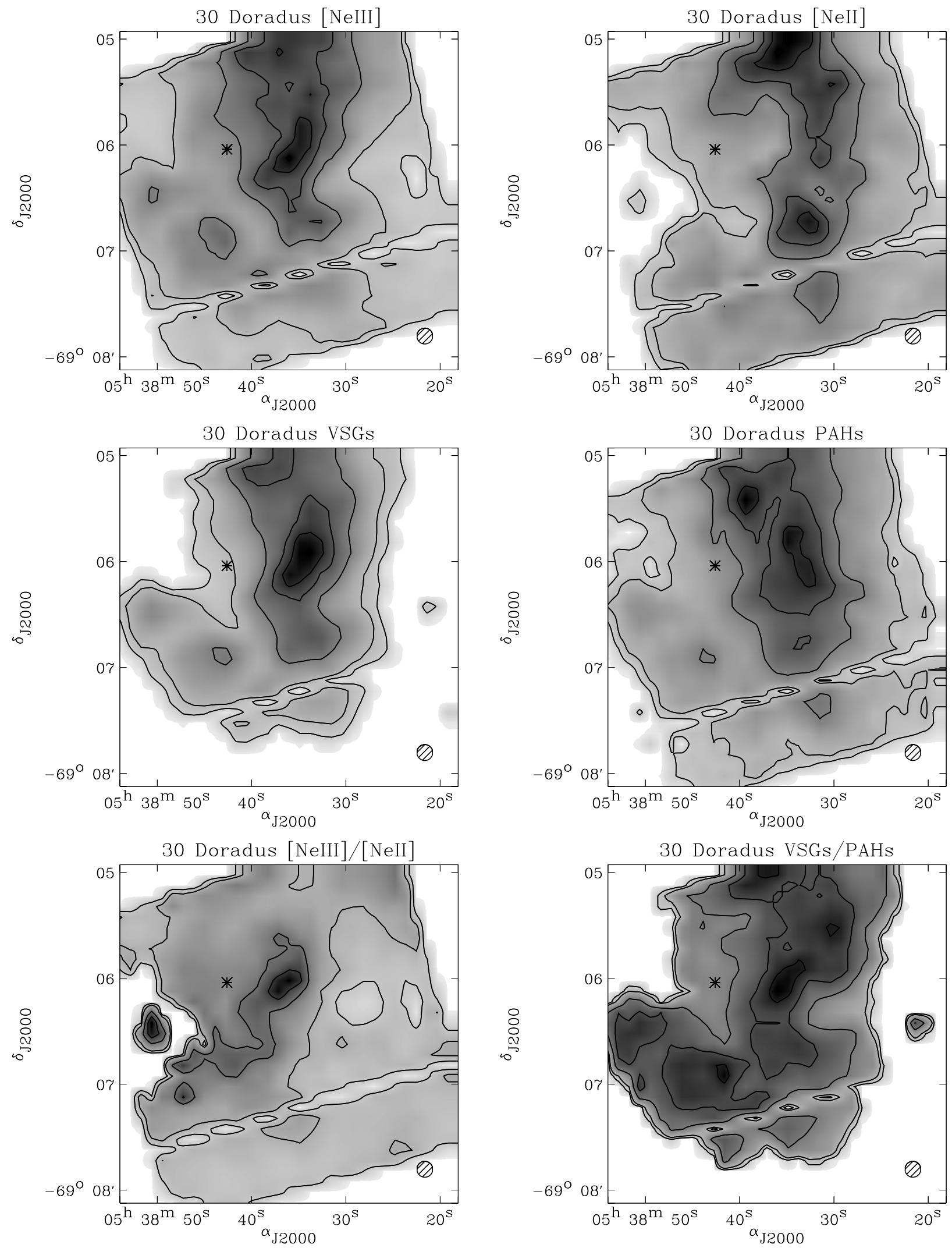

Fig. 6. Maps of some physical MIR components for 30 Dor. Up-left: [Ne III]; up-right: [Ne II]; middle-left: $15 \mu \mathrm{m}$ VSG continuum; middleright: PAHs; lower-left: [Ne III]/[Ne II]; lower-right: VSGs/PAHs; The contour levels are 5, 10, 30, 50, 70 and $80 \%$ of the peak intensity. The peak intensities are: $[\mathrm{Ne}$ III $]=1.6 \times 10^{-14} \mathrm{~W} \mathrm{~m}^{-2} \mathrm{beam}^{-1}$, [Ne II $]=3.5 \times 10^{-15} \mathrm{~W} \mathrm{~m}^{-2} \mathrm{beam}^{-1}, I(\mathrm{VSG})=7.2 \times 10^{-13} \mathrm{~W} \mathrm{~m}^{-2} \mathrm{beam}^{-1}$, $I(\mathrm{PAH})=1.4 \times 10^{-13} \mathrm{~W} \mathrm{~m}^{-2} \mathrm{beam}^{-1},[\mathrm{Ne} \mathrm{III}] /[\mathrm{Ne} \mathrm{II}]=34, I(\mathrm{VSG}) / I(\mathrm{PAH})=8.6$. The resolution has been degraded to the beam size at $16 \mu \mathrm{m}$ which is roughly $10^{\prime \prime}(2.4 \mathrm{pc})$. The strip across the lower part of the image is the inactive column 24 of ISOCAM. The asterisk marks the position of the stellar cluster R136. 
Table 4. Contribution of the different physical components for the global galaxies in various ISOCAM broadbands. The percentage is the fraction of the component compared to the total flux in the band (Table 3).

\begin{tabular}{|c|c|c|c|c|c|c|c|c|c|c|c|}
\hline & \multicolumn{2}{|c|}{$\overline{\text { LW2 }}$} & \multicolumn{4}{|c|}{$\overline{\text { LW3 }}$} & \multicolumn{5}{|c|}{ LW10 } \\
\hline & PAHs & VSGs & [Ne III] & [Ne II $]$ & PAHs & VSGs & {$[\mathrm{Ne}$ II $]$} & [S IV] & [Ar III] & PAHs & VSGs \\
\hline NGC 1140 & $75 \%$ & $24 \%$ & $8.6 \%$ & $4.0 \%$ & $5.5 \%$ & $75 \%$ & $3.0 \%$ & $1.3 \%$ & $\ldots$ & $23 \%$ & $73 \%$ \\
\hline NGC 1569 & $95 \%$ & $3 \%$ & $11 \%$ & $1.4 \%$ & $6.3 \%$ & $80 \%$ & $1.6 \%$ & $4.6 \%$ & $0.6 \%$ & $29 \%$ & $63 \%$ \\
\hline NGC 5253 & $42 \%$ & $56 \%$ & $2.9 \%$ & $\ldots$ & $\ldots$ & $95 \%$ & $\ldots$ & $\ldots$ & $\ldots$ & $\ldots$ & $95 \%$ \\
\hline II Zw 40 & $50 \%$ & $49 \%$ & $3 \%$ & $\ldots$ & $\ldots$ & $96 \%$ & $\ldots$ & $6.3 \%$ & $0.9 \%$ & $1 \%$ & $94 \%$ \\
\hline 30 Dor & $88 \%$ & $11 \%$ & $5.9 \%$ & $1.1 \%$ & $3.5 \%$ & $89 \%$ & $1.3 \%$ & $7.7 \%$ & $0.9 \%$ & $14 \%$ & $75 \%$ \\
\hline SMC N 66 & $85 \%$ & $9 \%$ & $7.8 \%$ & $\ldots$ & $2.6 \%$ & $85 \%$ & $\ldots$ & $11 \%$ & $1.8 \%$ & $18 \%$ & $68 \%$ \\
\hline SMC B\#1 & $97 \%$ & $\ldots$ & $\ldots$ & $4.2 \%$ & $49 \%$ & $43 \%$ & $6.5 \%$ & $4.6 \%$ & $\cdots$ & $88 \%$ & $0.9 \%$ \\
\hline M 82 & $97 \%$ & $0.4 \%$ & $0.7 \%$ & $4.6 \%$ & $26 \%$ & $68 \%$ & $4.0 \%$ & $0.5 \%$ & $\ldots$ & $59 \%$ & $36 \%$ \\
\hline
\end{tabular}

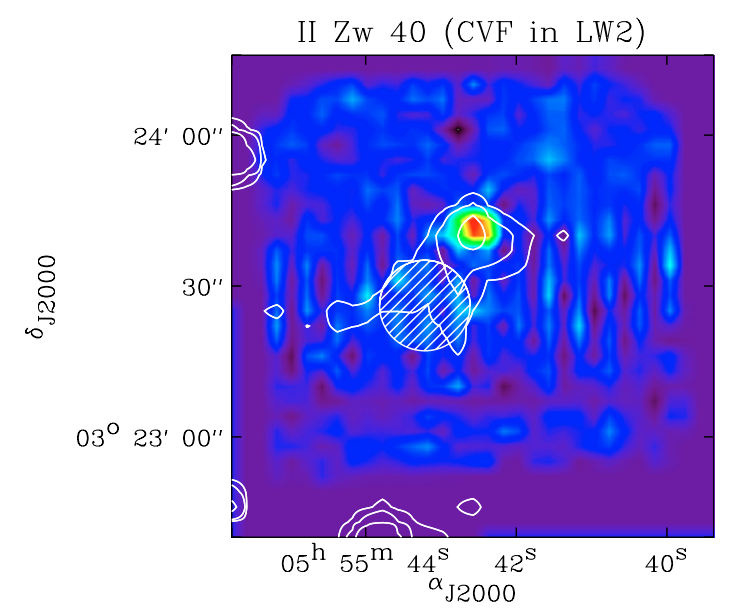

Fig. 7. The image is the result of the CVF spectral image of II $\mathrm{Zw} 40$ integrated in the equivalent LW2 band; contours are the R band image of Gil de Paz et al. (2003) which shows the prominent merger tail. The hatched $18^{\prime \prime}$ circle is the region of the CVF image that was integrated, resulting in the CVF spectrum in Fig. 1 (upper right spectrum). This region shows evidence for some faint PAH emission. The upper left spectra of Fig. 1 is integrated in a 14" circle toward the central MIR peak.

studied here, except SMC B\#1. In the active starburst galaxies, which globally resemble H II regions, NGC 5253 and II $\mathrm{Zw} 40$, almost all of the LW10 IRAS $12 \mu \mathrm{m}$ flux is due to the VSG emission. In the sources dominated by the disk or diffuse ISM, such as M 82 and SMC B\#1, the PAHs are the more important contributors with almost $90 \%$ of the $12 \mu \mathrm{m}$ broad band flux originating from PAHs. The [S IV] $\lambda 10.51 \mu \mathrm{m}$ is $5 \%$ to $9 \%$ of the LW10 band flux in the H II regions, 30 Dor and SMC N 66 and in the low-metallicity H II region-like galaxies, NGC 1569 and II Zw 40. With these beam sizes, the ionic lines in M 82 are certainly small contributors to any of the broad bands - they are dwarfed by the prominent PAH bands and the VSGs.

In the nearby $(4.5 \mathrm{Mpc})$ spiral galaxy, M 83, which was well-mapped in many ISOCAM broad bands as well as the CVF, it was possible to isolate the spectra of the nuclear region, the spiral arms, the interarm region and the diffuse, extended ISM (Vogler et al. 2005). While PAHs are the dominant contribution to the $15 \mu \mathrm{m}$ LW3 broad band flux in the diffuse ISM and the galactic disk, they are responsible for no more than $60 \%$ of the nuclear flux. Toward the nucleus, the PAH bands comprise most of the LW2 broad band flux in M 83, since the starburst region itself is not resolved by the ISOCAM beam, which is, instead, tracing the circumnuclear material. Globally, the MIR emmission in M 83, as well as other spiral galaxies, is dominated by the disk material (e.g., PAH bands), not the nucleus (e.g. Roussel et al. 2001b; Dale et al. 2001; Vogler et al. 2005). In contrast, the component contribution to the global broad bands of the dwarf galaxies sampled here typically have MIR characteristics similar to those of the nuclei of spiral and starburst galaxies, except for the fact that the abundance of PAH bands is much lower in these low metallicity sources. The dearth of dominant disk-emitting material is evident in the MIR spectra.

\subsection{Very hot $5.5 \mu \mathrm{m}$ dust}

Inspection of individual spectra throughout the galaxies, zooming into the short wavelength end of the ISOCAM spectra, reveals that the continua usually decreases to near zero value at the beginning of the ISOCAM CVF spectra at $5.5 \mu \mathrm{m}$, considering the uncertainty of the measurements. Isolated regions of NGC 1569 (Fig. 8) and 30 Dor (Fig. 9), however, are the exceptions. These regions show excess at 4.9 to $5.6 \mu \mathrm{m}$ which could either be due to very hot dust or the stellar population. While the detailed modelling of the stellar population and gas and dust of low metallicity galaxies demonstrates that the stellar spectra are dominated by younger stellar populations on global scales, usually less than 10 Myr (e.g. Galliano et al. 2003, 2005), there can also be some contribution, on global scales to the stellar continuum toward shorter wavelengths in low metallicity dwarf galaxies, but usually more prominant at wavelengths shorter than those traced by the ISOCAM CVF (e.g. Engelbracht et al. 2005).

Figure 10 shows the spatial distribution of the continuum between 4.9 and $5.6 \mu \mathrm{m}$ in NGC 1569 overlayed on the $\mathrm{H} \alpha$ map by Waller (1991). The 4.9 to $5.6 \mu \mathrm{m}$ emission is very localised and peaks at the site of the brightest $\mathrm{H} \alpha$ peak, which is in close proximity to a super star cluster. The $5.5 \mu \mathrm{m}$ emission falls off rapidly, suggesting an origin of very localised, hot dust emission. It is also spatially associated with the [Ne III] and VSG peaks, and not necessarily related to the PAHs (Fig. 5). 


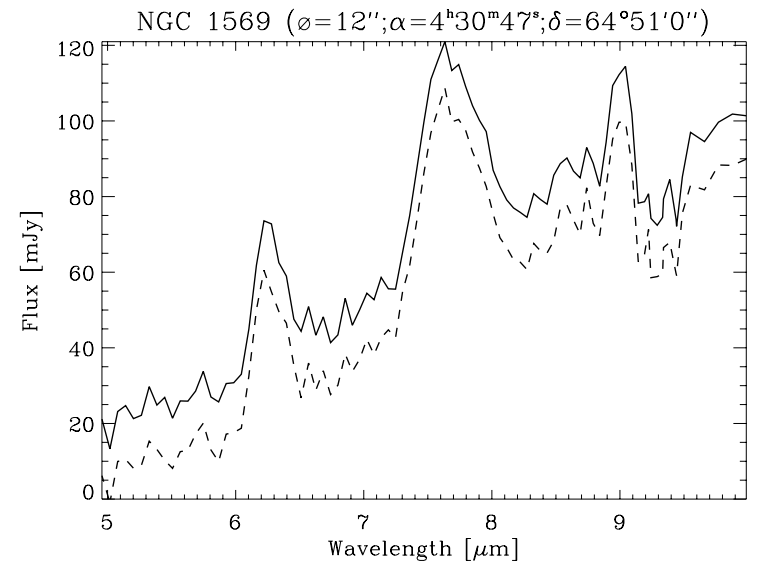

Fig. 8. A zoom into the short wavelength part of the CVF spectrum of NGC 1569 toward the central $12^{\prime \prime}$ of the peak (Fig. 10). (solid line). The dashed line represents the lower limit of this spectra, taking into account the errors $\left(F_{v}-\Delta F_{v} / 2\right)($ Sect. 2).

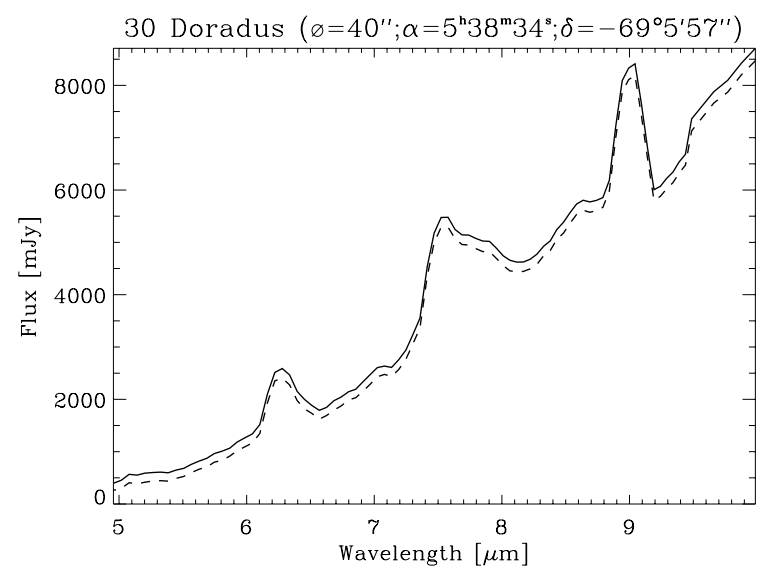

Fig. 9. Zoom on the short wavelength part of the global CVF of 30 Dor (solid line). The dashed line represents the lower limit of this spectra, taking into account the uncertainty $\left(F_{v}-\Delta F_{v} / 2\right)$ Sect. 2).

Likewise, toward the 30 Dor region, the 4.9 to $5.6 \mu \mathrm{m}$ continuum is very localised (Fig. 11), peaking toward the [Ne III] and VSG peak, about $10 \mathrm{pc}$ from the R136 cluster (Fig. 11). Hot dust emission at these wavelengths has been inferred in other starburst galaxies (Hunt et al. 2002). If dust is emitting at these short wavelengths, the dominant particles must be small (nanometer sizes) and stochastically heated with temperature fluctuations as high as $\simeq 1000 \mathrm{~K}$ (Guhathakurta \& Draine 1989).

\section{Interpretation}

Using the spectral fitting procedure described above (Sect. 3.2), the global ratios of the physical MIR components, the nebular lines, the PAHs and the VSGs have been computed. In this section we present and study relationships between these components in our various sources and provide interpretation to help elucidate the nature of the ISM of low metallicity environments.

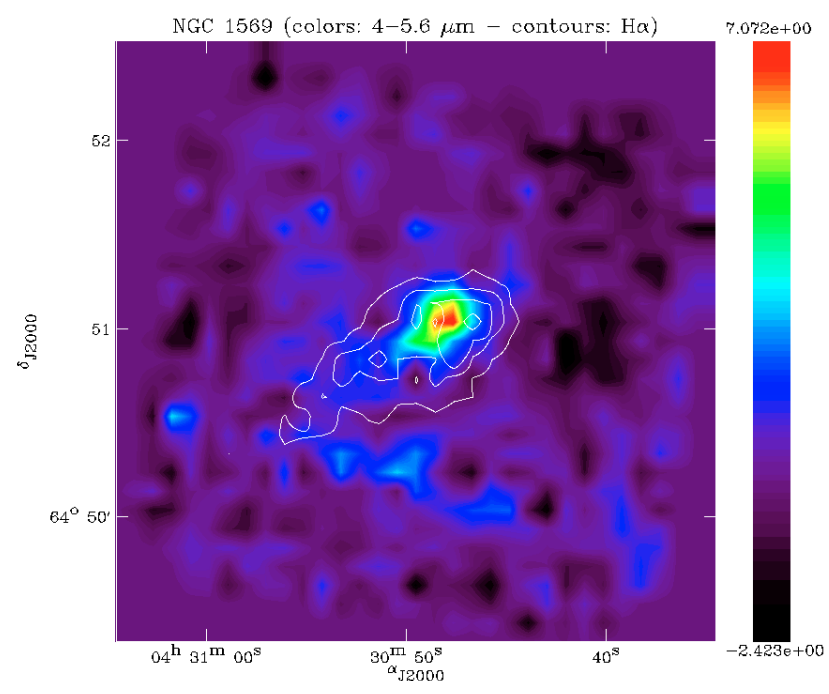

Fig. 10. Very hot dust emission. The image is the integration of the CVF spectrum of NGC 1569 from 4.9 to $5.6 \mu \mathrm{m}$. Contours are from the H $\alpha$ map of Waller (1991).

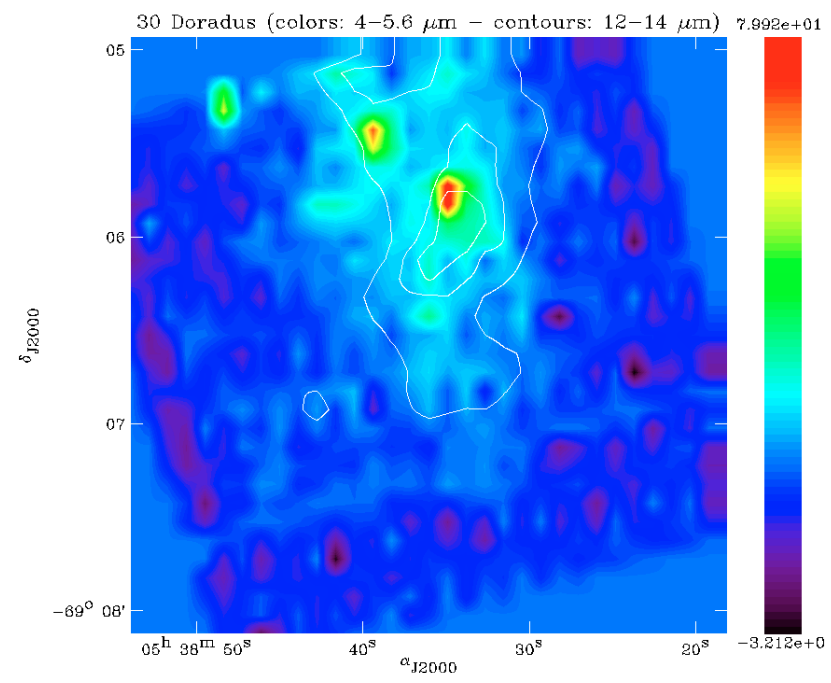

Fig. 11. Very hot dust emission. This map is the integration of the CVF spectrum of 30 Dor from 4.9 to $5.6 \mu \mathrm{m}$. Contours are the VSG distribution, which also resembles that of the [Ne III] $\lambda 15.56 \mu \mathrm{m}$ (see Fig. 6).

\subsection{The PAH bands}

One of the most striking aspects of the spectra of these low metallicity starburst systems is the dearth of PAH emission along with the steeply-rising VSG continuum beyond $10 \mu \mathrm{m}$. This is a very unusual circumstance, since the photons which produce the elevated temperatures of the VSG continuum would also illuminate the PAHs, if they were present.

This consistent discrepency of the paucity of bright PAH bands along with elevated continuum in the MIR spectra of a sample of low metallicity galaxies was demonstrated with ISOCAM spectro-imaging in Thuan et al. (1999); Madden (2000, 2005). Ground-based observations from 1991, already showed that PAHs were not prominent in 2 dwarf galaxies (Roche et al. 1991). Recent Spitzer observations also confirm this (Houck et al. 2004; Engelbracht et al. 2005; 


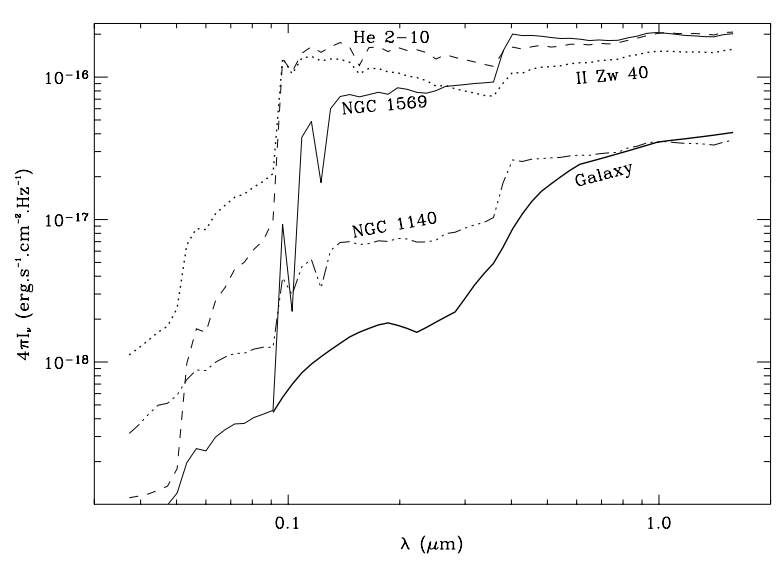

Fig. 12. The variations of the modeled ISRFs for 4 of the dwarf galaxies compared to the Galaxy (taken from Galliano et al. 2003, 2005).

Wu et al. 2005). Dustier starbursts or spiral galaxies, on the other hand, do show prominent PAHs in their MIR spectra, even on global scales (e.g. Genzel et al. 1998; Laurent et al. 2000; Förster Schreiber et al. 2003; Vogler et al. 2005). There can be several reasons for the relatively low PAH intensity seen here: a) the PAHs are destroyed globally in mow-metallicity systems via evaporation and photodissociation processes, due to radiation field properties, such as hardness and intensity (e.g. Madden 2000, 2005); b) the PAHs are present but only on small spatial scales, which may be a consequence of (a). In this case, their emission could be suffering from dilution due to the telescope beam, and could be more apparant on smaller spatial scales; c) PAHs were never a chemically abundant species in these galaxies, due to their low metalliciy nature.

We begin to investigate effects due to the properties of the radiation field by first comparing the intrinsic ISRFs of 4 dwarf galaxies, as modeled by Galliano et al. (2003, 2005). As can been seen in Fig. 12, the global intrinsic ISRFs of the dwarf galaxies are all harder and more intense relative to the global ISRF of the Galaxy. For example, the global ISRF of NGC1140, where the MIR spectra do indeed show evidence for relatively prominent PAH bands, is obviously softer, and less intense than those of II Zw 40 and NGC 1569, where the PAH bands are less prominent. We look more closely into this quantitatively in the following sections.

\section{2. [Ne III]/[Ne II] line ratios: effects on the survival of PAHs?}

The diagnostic value of the MIR nebular line ratios, particularly that of the $[\mathrm{Ne}$ III $] \lambda 15.56 \mu \mathrm{m}$ and the $[\mathrm{Ne}$ II $] \lambda 12.81 \mu \mathrm{m}$ lines, has received a great deal of attention lately, initiated by the abundant ISO spectra in a wide variety of sources. The ionisation potentials of neutral $\mathrm{Ne}$ and $\mathrm{Ne}^{+}$are 21.5 and $40.9 \mathrm{eV}$, respectively. The ratio of the $[\mathrm{Ne} \mathrm{III}] /[\mathrm{Ne}$ II] fine structure lines are independent of the neon abundances and are relatively independent of extinction, since their wavelengths are not very different. They are sensitive to the ionisation structure of the nebulae and to the spectral form of the UV radiation field, thus, they trace populations of massive hot stars, characterised by their hard radiation fields. Deciphering the precise ionisation

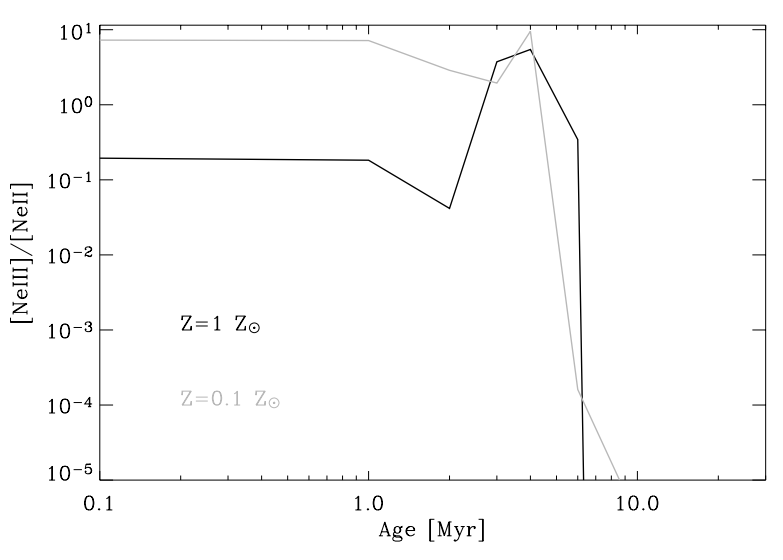

Fig. 13. The behavior of the $[\mathrm{Ne}$ III] $] /[\mathrm{Ne}$ II] line ratio as a function of age for clusters of solar metallicity (black curve) and 0.1 solar metallicity (grey curve). A single instantaneous starburst was assumed with a Salpeter IMF.

structure of nebulae from ionic line ratios involves untangling the intertwined effects of metallicity, morphology, spectral type and temperature to finally arrive at the stellar composition and age - important characteristics for studying the evolution of starbursts within galaxies and from galaxy to galaxy. This is not completely straightforward.

The diagnostic value of the $[\mathrm{Ne}$ III $] /[\mathrm{Ne}$ II $]$ line ratios can be seen in Fig. 13, for solar and 0.1 solar metallicities. These examples are shown for a star cluster with a Salpeter IMF (upper mass cut-off, $m_{\text {up }}$, of $120 M_{\odot}$; lower mass cut-off, $m_{\text {low }}$, of $\left.0.1 M_{\odot}\right)$. The line ratio is boosted to ratios of the order of 10 to 100 between 1 to $3 \mathrm{Myr}$, due to contribution from the O stars. After this time, the $\mathrm{O}$ stars have moved off the main sequence and the $[\mathrm{Ne} \mathrm{III]} /[\mathrm{Ne} \mathrm{II}]$ line ratios plummet to very low values.

Decreasing the metallicity has effects on the $[\mathrm{Ne}$ III]/[Ne II] line ratios due to reduced line blanketing and blocking in the stellar atmospheres. For a given stellar type, the main sequence temperatures are hotter and the form of the stellar SED is harder. Metallicity effects on ionic line ratios have been studied by a number of authors (e.g. Thornley et al. 2000; Martín-Hernández et al. 2002b; Giveon et al. 2002; Rigby \& Rieke 2004). The [Ne III]/[Ne II] line ratios in low metallicity regions are strikingly high, often 1 to 2 orders of magnitude greater than more metal-rich starburst galaxies as demonstrated by Madden (2005). The outward increase of Galactic $[\mathrm{Ne} \mathrm{III}] /[\mathrm{Ne} \mathrm{II}]$ line ratios has also been attributed to the decrease in metallicity (Giveon et al. 2002).

Thornley et al. (2000) studied a large sample of starburst galaxies, and found relatively low [Ne III]/[Ne II] line ratios observed in solar metallicity starburst galaxies $(\leq 0.8)$. One interpretation could be a lack of massive stars $\left(\gtrsim 50 M_{\odot}\right)$, but this would be difficult to reconcile given other evidence for massive stars. Their favoured explanation for low [Ne III]/[Ne II] line ratios was the aging of relatively short-lived $\left(10^{6}\right.$ to $\left.10^{7} \mathrm{yr}\right)$ starbursts (see also Rieke et al. 1993; Engelbracht et al. 1998). In this scenario, the hottest stars are not necessarily the dominant source of the ionisation in the starburst galaxy, accounting for the low line ratio values. Another scenario proposed for the low $[\mathrm{Ne}$ III $] /[\mathrm{Ne}$ II] line ratio observed in metal-rich starburst 
Table 5. Ionic line intensity ratios for the low metallicity galaxies (global values).

\begin{tabular}{lllll}
\hline \hline & {$[\mathrm{Ne}$ III]/[Ne II] } & {$[\mathrm{S} \mathrm{IV}] /[[\mathrm{Ne}$ III $]$} & {$[\mathrm{Ar} \mathrm{III}] /[\mathrm{Ne}$ III $]$} & I(PAH)/[(VSG) \\
\hline NGC 1140 & $2.2 \pm 0.7$ & $0.24 \pm 0.11$ & $\lesssim 0.01$ & $1.33 \pm 0.37$ \\
NGC 1569 & $7.7 \pm 1.7$ & $0.45 \pm 0.11$ & $0.09 \pm 0.05$ & $1.20 \pm 0.22$ \\
NGC 5253 & $\gtrsim 11$ & $\lesssim 0.25$ & $\lesssim 0.05$ & $\lesssim 0.30$ \\
II Zw 40 & $\gtrsim 5.7$ & $3.53 \pm 0.65$ & $\lesssim 1.5$ & $\lesssim 0.88$ \\
30 Dor & $5.59 \pm 0.41$ & $1.29 \pm 0.10$ & $0.25 \pm 0.02$ & $0.36 \pm 0.01$ \\
SMC N 66 & $\gtrsim 10.3$ & $1.59 \pm 0.18$ & $0.36 \pm 0.09$ & $1.4 \pm 0.6$ \\
SMC B\#1 & $\lesssim 0.5$ & $\gtrsim 1.7$ & $\ldots$ & $\gtrsim 56$ \\
M 82 & $0.147 \pm 0.012$ & $1.09 \pm 0.10$ & $\lesssim 0.4$ & $3.78 \pm 0.11$ \\
\hline
\end{tabular}

galaxies is that the massive stars could spend most of their main sequence lifetimes concealed in very dusty, extincted regions, undetected even out to MIR wavelengths (Rigby \& Rieke 2004).

The nebular line intensities for our low metallicity sources are listed in Table 3 while the line ratios are shown in Table 5. We also include M 82 for comparison with a metal-rich starburst galaxy. Except for M 82 and the diffuse, low metallicity region, SMC N 66, all of the other sources show $[\mathrm{Ne} \mathrm{III}] /[\mathrm{Ne} \mathrm{II}]$ line ratios $>2$ and as high as 15 or more averaged over full galaxy sizes. Note that the lower limits on the $[\mathrm{Ne}$ III $] /[\mathrm{Ne}$ II $]$ ratios are due to the low signal-to-noise of the [NeII] line. Many of these starburst regions are known to be harbouring super star clusters (SSCs) and the modeled ISRFs for some of these sources is obviously much harder than that of the Galaxy, for example (Fig. 12).

Not only are the $[\mathrm{Ne} \mathrm{III}] /[\mathrm{Ne} \mathrm{II}]$ line ratios very high within the resolved galaxy NGC 1569 (Sect. 3.3.1; Fig. 16), but high averaged $[\mathrm{Ne} \mathrm{III}] /[\mathrm{Ne} \mathrm{II}]$ line ratios are also observed on averaged full galaxy scales for the more distant, unresolved galaxies (Table 5). To account for an averaged high $[\mathrm{Ne} \mathrm{III}] /[\mathrm{Ne} \mathrm{II}]$ line ratio in the unresolved galaxies, the ionising photons would need to be present in a relatively large area of the galaxy (Madden 2000, 2005). One possibility would be an ISM that is very porous, allowing the ionising radiation to penetrate the galaxy on large scales (more on this hypothesis in Sect. 4.3).

To investigate the effect of the hard radiation fields on the PAHs, we inspect the behavior of the $[\mathrm{Ne}$ III] $/[\mathrm{Ne} \mathrm{II}]$ and PAH/VSGs for our dwarf galaxies (Fig. 14), first demonstrated in (Madden 2005). For comparison, we also include many other sources such as the H II region sample of Peeters et al. (2002), starburst galaxies from the ISOCAM sample of Laurent et al. (2000) and spiral galaxies from the sample of Roussel et al. (2001a). The MIR spectra were modeled and the various components were extracted as described in Sect. 3.2. The PAH quantity presented here is the sum of the fluxes the 5 PAH bands ${ }^{1}(\lambda=6.2,7.7,8.6,11.3$, $12.6 \mu \mathrm{m}$ ) in our CVF spectra. Using all of the features to measure the behavior of the PAHs as opposed to a single feature, avoids the known effects of feature-to-feature ratio variations, which can be very noticable under widely varying UV environments (e.g. Vermeij et al. 2002; Galliano 2004). The quantity

\footnotetext{
${ }^{1}$ PAH bands refer to the features extracted in the manner described in Sect. 3.2, without the continuum from the VSGs.
}

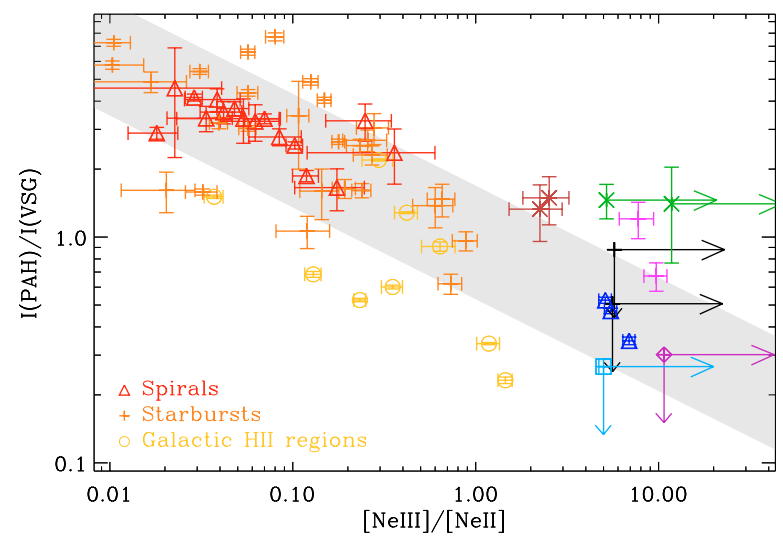

Fig. 14. Correlation between $[\mathrm{Ne} \mathrm{III}] /[\mathrm{Ne} \mathrm{II}]$ and $15 \mu \mathrm{m} / \mathrm{PAH}$, for H II regions (Peeters et al. 2002), spiral, starburst and dwarf galaxies (Madden 2005). The dwarf galaxies are located toward the right of the plot (see Fig. 15 for the dwarf galaxy color code). The grey strip is the linear fit to the spread of points $\pm 1 \sigma$. The arrows on the dwarf galaxy values indicate measurement limits which are limited by the low detection levels of the [Ne II] $\lambda 12.81 \mu \mathrm{m}$ and the PAHs. See the electronic version of the Journal for color version of the plots.

characterising the VSG emission is the fitted, feature-free continuum of the MIR spectra (Sect. 3.2), integrated between 10 and $16 \mu \mathrm{m}$. Notice the variations of 3 orders of magnitude of the observed [Ne III]/[Ne II] line ratios. Metal-rich starburst regions typically show a range of low excitation values of about 0.01 to 0.15 (see also Thornley et al. 2000). The ratio of the $[\mathrm{Ne} \mathrm{III}] /[\mathrm{Ne}$ II $]$ lines in $\mathrm{H}$ II regions range from 0.1 to 1.0 and the low-metallicity sources show the highest values up to and beyond ratios of 10 . The large values of [Ne III]/[Ne II] line ratios are indicative of the hard ISRFs within the low-metallicity regions.

We find a correlation which indicates that as the hardness of the radiation field increases, as traced by the high $[\mathrm{Ne}$ III]/[Ne II] line ratios, the ratio of PAH/VSG decreases.

Figure 15 is a zoom into Fig. 14, showing the low metallicity sources, which occupy the higher values of the $[\mathrm{Ne}$ III $] /[\mathrm{Ne}$ II] line ratios. As another check, we quantify the hardness of the ISRFs directly in Fig. 12 by:

$$
\frac{\int_{0}^{\infty} I_{v} \mathrm{~d} v}{\int_{0}^{\infty} \frac{I_{v}}{h v} \mathrm{~d} v}
$$




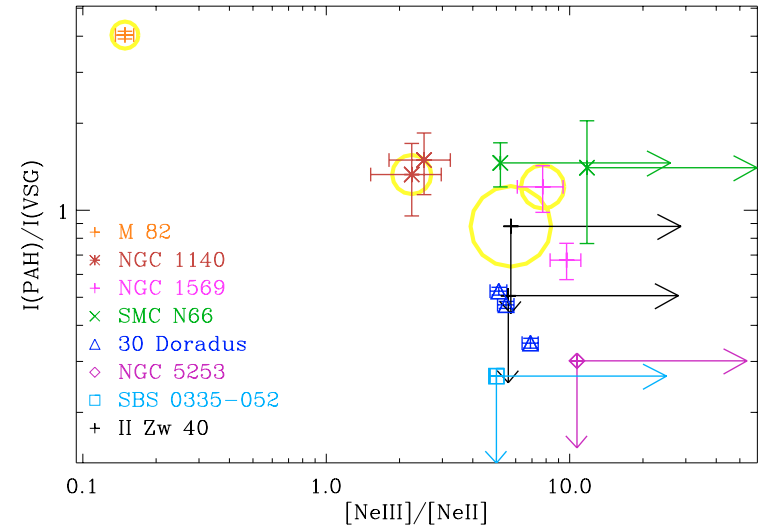

Fig. 15. Correlation between $[\mathrm{Ne} \mathrm{III}] /[\mathrm{Ne} \mathrm{II}]$ and $\mathrm{PAH} / \mathrm{VSG}$, for global values of dwarf galaxies, and M 82 for contrast (a zoom into Fig. 14). The $[\mathrm{Ne} I I I] /[\mathrm{Ne} I I]$ value for SBS $0335-052$ is from Houck et al. (2004). For some well-resolved galaxies we also include a central, smaller region. The size of the circles around some sources represents the relative hardness of the modeled ISRFs (Fig. 12), as described by equation 2. For example, the larger the circle, the harder the intrinsic ISRF. The arrows indicate measurement limits which are limited by the low detection levels of the [Ne II] $\lambda 12.81 \mu \mathrm{m}$ and the PAHs. See the electronic version of the Journal for color version of the plots.

This ratio, representing the relative hardness of the ISRF of the galaxies, is schematically represented by the size of the circles in Fig. 15. We see that the relative hardness of the ISRF increases as the ratio of PAH/VSG decreases, consistent with the increasing $[\mathrm{Ne}$ III] $/[\mathrm{Ne}$ II] line ratios.

Thus, both the increasing [Ne III]/[Ne II] line ratios, measured from the MIR spectra, as well as the hardness of the intrinsic ISRF, measured from the modeled SEDs, show a striking increase as the PAH/VSG decreases. Thus, this is consistent with the the hard radiation field being responsible for the destruction of the PAHs, and the steeply rising continuum emission from the hot, small grains.

In Fig. 16, we plot the values of the $[\mathrm{Ne} \mathrm{III}] /[\mathrm{Ne}$ II] line ratios and PAH/VSG for individual pixels within NGC 1569, 30 Dor, SMC N 66, as well as the metal-rich starburst and spiral galaxies, M 82 and M 51, NGC 6946, M 83 and NGC 1365. A spread of the $[\mathrm{Ne}$ III]/[Ne II] line ratios of about 1 and up to 2 orders of magnitude exists within these sources, while the values of the ratios of the PAH/VSG span over a range of about 5 to 10 within sources. This plot also demonstrates that the 3 well-resolved low metallicity sources have high $[\mathrm{Ne}$ III]/[Ne II] line ratios even from point-to-point within the galaxies, confirming the galaxy-wide spatial extent of the hard radiation field see throughout NGC 1569 (Sect. 3.3). The more metal-rich galaxies never have exceptionally high values of $[\mathrm{Ne}$ III]/[Ne II] line ratios nor low PAH/VSG values. While metallicity gradients can be an explanation for some of the spread in the dustier galaxies, it can not be the explanation for NGC 1569, 30 Dor and SMC N 66. SMC N 66 and 30 Dor are confined H II and PDR regions that can not vary significantly in metallicity and NGC 1569, like other low metallicity galaxies, is not known to have a metallicity gradient (Kobulnicky \& Skillman 1997). We also confirm that within these sources the largest values of the $[\mathrm{Ne} \mathrm{III}] /[\mathrm{Ne}$ II] line ratios coincide with the

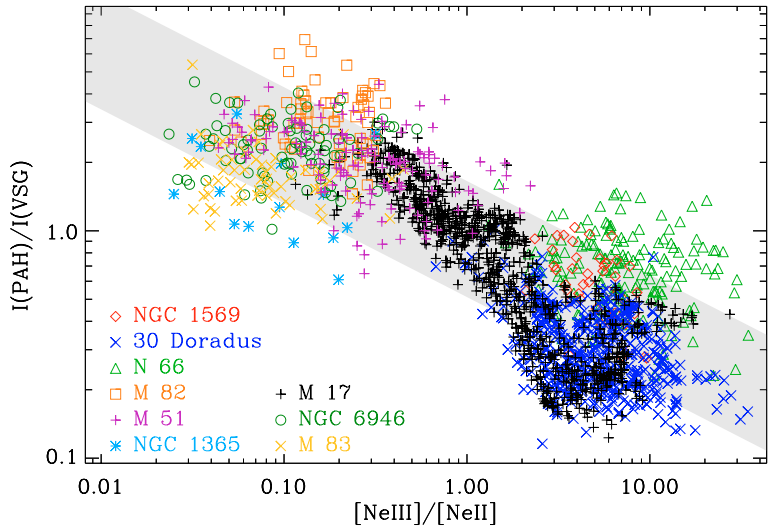

Fig. 16. Correlation between $[\mathrm{Ne}$ III]/[Ne II] and PAH/VSG for regions within resolved sources, such as the low metallicity sources NGC 1569, 30 Dor and SMC N 66. Also included for comparision are regions within the more metal-rich galaxies, M 51, M 82, NGC 6946, M 83 and NGC 1365. Note that the metal-poor objects occupy a spread of values located to the lower right of the plot, while the more metalrich objects have lower $[\mathrm{Ne} \mathrm{III}] /[\mathrm{Ne} \mathrm{II}]$ ratios and higher $\mathrm{PAH} / \mathrm{VSG}$ line ratios. The grey strip is from Fig. 14. See the electronic version of the Journal for color version of the plots.

prominent H II regions/SSCs within the object, and decrease outward. Thus the hardness of the stellar radiation does appear to be traced here by the $[\mathrm{Ne}$ III] $/[\mathrm{Ne}$ II] line ratios, and may control the destruction of the PAHs within galaxies.

\subsection{PAH destruction mechanisms}

PAHs are known to be mostly absent from H II regions. The MIR spectra of AGNs in contrast to those of starburst galaxies, was already noted to be void of PAH features in pioneering work 20 years ago (Roche \& Aitken 1985; Aitken \& Roche 1985) and also demonstrated more recently in Siebenmorgen et al. (2004). Most explanations follow on the suggestion of the destruction of PAHs being due to the hard radiation field of the central source. The heating by energetic photons can basically, either ionise or dissociate PAHs. However, the precise processes and environmental factors involved, and how this varies as a function of the chemical properties such as the state of ionisation and the size of the molecule, continues to be a subject of investigation (e.g. Voit 1992; Jochims et al. 1994; Joblin et al. 1995; Allain et al. 1996a,b; Allamandola et al. 1999; LePage et al. 2003). The apparant lack of small PAHs ( $<20$ atoms) in the ISM (e.g. Clayton et al. 2003) suggests that such small molecules are readily destroyed under widely varying ISM conditions. Depending on photon energies, critical sizes for dehydrogenating PAHs can be 20-30 atoms (LePage et al. 2003) and even larger ones can be ionised. The derrived threshold size of the PAH molecule depends on the model used.

Recent high spatial resolution MIR observations of 2 starburst galaxies by Tacconi-Garman et al. (2005) also show the PAH and continuum emission peaking at star formation sites, while an obvious suppression of the PAH feature to continuum occurs specifically toward the star formation peaks. Their investigatigation suggests photoionisiation and/or 
photodissociation of PAHs as plausible mechanisms to explain the observations.

The fact that PAHs are not present in Galactic H II regions and the PAH features to continuum ratio decreases toward the star formation peaks (Fig. 5), and the effect of the overall tendency for the PAH to continuum ratios to decrease as the $[\mathrm{Ne} \mathrm{III}] /[\mathrm{Ne} \mathrm{II}]$ line ratios increase, are consistent with the conclusion that the lack of PAHs observed in dwarf galaxies is due to the hardness of the ISRF.

\subsection{Other factors possibly playing a role in the observed low PAH intensities}

The possibility for low-metallicity systems to chemically, have low abundances of PAHs must also be explored. Taking into account possible ISM enrichment sources for carbonaceous particles, such as AGB stars and/or supernovae Type II (SN II), and not considering PAH destruction processes, we can eliminate SN II as large contributors of PAHs, due to their relatively short life times. The galaxies considered would already have experienced mass enrichment from SN II. Dwek (2005); Galliano \& Dwek (2005) have considered this issue and note that AGB stars begin to contribute to the carbon reservoir only when $4 M_{\odot}$ stars have entered the AGB stage $\left(5 \times 10^{8} \mathrm{yr}\right)$. Assuming AGB stars are the dominant source of PAHs, this could explain why PAHs are not abundant in extremely low metallicity systems $(<0.1$ solar $)$. For the more moderate metallicity systems considered here, PAHs should indeed be present, under this hypothesis. If, on the other hand, SN II were to be a dominant source of PAHs, then PAHs should likewise be present in these systems, if destruction were not taken into account. Note that model results can depend strongly on the adopted yields (e.g. Dwek 2005). Photodestruction of PAHs as well as chemistry effects are probably both playing important roles in low-metallicity systems.

Figure 17 shows the observed PAH/VSG as a function of the metallicity for the sources considered here. There does appear to be a correlation related to the relative intensity of PAH bands and the metallicity of the object, such that as the metallicity decreases, the PAH/VSG ratio decrease. The range of metallicities that we consider here is limited. However, Engelbracht et al. (2005) find such a correlation using broad band observation for a wide range of low metallicity galaxies.

What are the chances of us missing compact photodissociation regions, where PAHs could be residing in very small clumps and which could be suffering from significant beam dillution? The ISOCAM beam at the shorter wavelengths, is about $6^{\prime \prime}$, which is about $500 \mathrm{pc}$ for the most distant source in our study (NGC $1140 \sim 23 \mathrm{Mpc}$ ). The fact that we see quite the pervasive ionic gas in the ISM over galaxy-wide scales in resolved galaxies such as NGC 1569, as well as on the global scales of unresolved galaxies, supports the idea of a very porous ISM where the mean free path length of the ionising photons can be relatively large due to the decrease in dust attenuation. The fact that the FIR fine structure lines, such as the important cooling line of $158 \mu \mathrm{m}$ [CII], are relatively bright in all of these galaxies, infers a substantial amount of dense

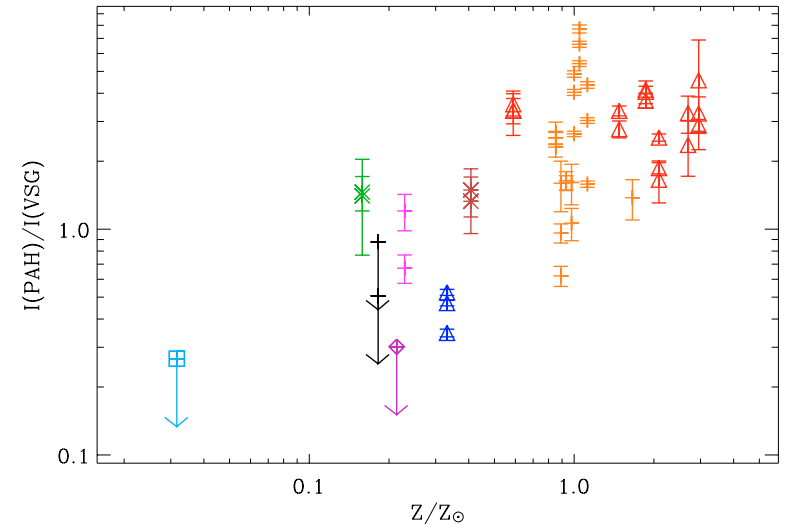

Fig. 17. Correlation between PAH/VSG and metallicity $\left(Z / Z_{\odot}\right)$. Symbols for the dwarf galaxies, starburst galaxies and Galactic H II region are those used in Figs. 14 and 15.

clumpy, small filling-factor PDR material (Madden 2000). In support of this hypothesis, to account for the observed FIR to submm SEDs in dwarf galaxies, Galliano et al. (2005) invoked a scenario of small dense clumps, with size scales on the order of a few tens of pc, having a small volume factor of $10^{-4}$ to $10^{-3}$. If this hypothesis were correct, then PAHs could indeed be hidden from our view in the relatively large ISOCAM beam. One example of this point is in NGC 5253, where the $15 \times 30^{\prime \prime}$ ISOSWS beam barely produced evidence for low level PAH bands (Fig. 1), while more recent ground-based observations by Alonso-Herrero et al. (2004) use a beam size comparable to 5pc in NGC 5253 with which they are able to detect the weaker PAH band at $3.3 \mu \mathrm{m}$. While this could be a possible explanation for the MIR spectra considered here, it would probably not support a significant mass of PAHs residing in these small clumps.

\section{Conclusion}

We present new ISOCAM MIR spectra of the low metallicity dwarf galaxies, NGC 1569, NGC 1140 and II Zw 40, along with MIR spectra from the 30 Dor region in the low metallicity LMC. These are compared with other published spectra of low metallicity regions as well as metal-rich starburst galaxies. The low metallicity nature of the ISM presents a different picture than that of dustier starburst galaxies or our own Milky Way. We summarise our findings:

- The characteristics of the ISM that dominate the MIR emission of low metallicity regions differ strikingly from those of dustier starburst or spiral galaxies. The most prominent aspect of the MIR spectra of low metallicity regions, at least down to the scale of the ISOCAM beam, is the dearth of PAH bands. Even in the full galaxy averaged spectra of these galaxies, the MIR characteristics are often dominated by steeply rising small grain continua and prominent ionic lines, most notably the [Ne III] and [S IV] lines, in contrast to dustier starburst galaxies, which globally show prominent emission arising from the disk component (i.e. PAHs and relatively flatter MIR continuum). The distribution of various ISM components in low metallicity 
environments appears to have been affected by the decrease in dust abundance, which effectively translates into a larger mean free path length of the UV photons. This may explain why the low metallicity galaxies tend to resemble giant $\mathrm{H}$ II regions viewed on large galactic scales, with smaller, clumpy PDRs interspersed within the ISM (e.g. Galliano et al. 2003, 2005).

- We quantify the contributions of ionic lines, continuua and PAH bands in various broad bands, which were often used to map galaxies. While the $12.7 \mu \mathrm{m}$ PAH band dominates the $15 \mu \mathrm{m}$ ISOCAM broad band emission on galaxy-wide scales spiral galaxies, this same broad band is typically dominated by the hot small grain continuum emission in low metallicity galaxies with up to $10 \%$ of the $15 \mu \mathrm{m}$ broad band emission arising from the $[\mathrm{Ne}$ III $] \lambda 15.56 \mu \mathrm{m}$ alone. In the $6.7 \mu \mathrm{m}$ broad band of ISOCAM, PAHs, even as faint as they are, dominate, in some cases. However, in the more active low metallicity galaxies such as NGC 5253 and II Zw 40, for example, this band captures 50\% PAH emission and $50 \%$ hot dust continuum emission. Thus caution must be used when interpreting broad band emission: low metallicity regions can have very different contributions to the MIR broad band emission compared to that of the more metal-rich starburst galaxies.

- NGC 1569 and 30 Dor are well resolved spatially and the distribution of the various tracers are inspected. 30 Dor images are the higest spatial resolution examples in our sample, with a resolution of $2.4 \mathrm{pc}$. The [Ne III] $\lambda 15.56 \mu \mathrm{m}$ emission is distributed in a sharp ridge closer to the R136 exciting star than the [Ne II] line emission. The PAH peaks avoid the more confined [Ne III]/[Ne II] peak, which is tracing the hard radiation field, while the VSG peak prefers the $[\mathrm{Ne}$ III]/[Ne II] peak. In NGC 1569, with the spatial resolution of $106 \mathrm{pc}$, most of the species, in general, appear to peak toward the star formation sites. In NGC 1569, the extents of the $[\mathrm{Ne}$ II $] \lambda 12.81 \mu \mathrm{m}$ and the $[\mathrm{Ne}$ III $] \lambda 15.56 \mu \mathrm{m}$ lines are remarkable, even tracing some of the $\mathrm{H} \alpha$ ejecta.

- We find high local [ $\mathrm{Ne} \mathrm{III}] /[\mathrm{Ne} \mathrm{II}]$ ratios throughout the resolved low metallicity galaxies, and likewise high values averaged over the unresolved galaxies, often as high as 10 - much higher than dustier starburst galaxies. Due to the lower dust abundance, hard UV photons presumably can permeate over larger size scales within galaxies. We find a prominent correlation between the PAHs/VSGs ratios and the $[\mathrm{Ne} \mathrm{III}] /[\mathrm{Ne}$ II] ratios for the low metallicity sources as well as Galactic H II regions and more metal-rich starburst galaxies. They span 3 orders of magnitude in the sample presented here. The correlation of the $[\mathrm{Ne} \mathrm{III}] /[\mathrm{Ne}$ II $]$ ratios and the PAHs/VSGs is also seen within sources which have sufficient spatial resolution. The hard, permeating radiation field seems to play an important role in the destruction of PAHs in low metallicity regions. It does not appear to be simply a matter of metallicity controlling the spectral characteristics of dwarf galaxies, but rather a combined complex effect of the intrinsic radiation field plus the low metallicity and, undoubtedly, the geometry of the sources and the structure and evolution of the ISM. MIR spectra from a broader sample of lower metallicity galaxies with a variety of star formation activity plus higher spatial resolution observations would help to shed more light on these issues.

Acknowledgements. A special thanks to Els Peeters for providing the H II region spectra. We thank René Gastaud, Pierre Chanial and Héléne Roussel for expert advice on various stages of the ISOCAM data reduction. We thank the anonymous referee for helpful comments that improved the clarity of the paper. This work was supported in part by a National Research Council Research Associateship for F. Galliano at NASA GSFC.

\section{References}

Aitken, D. K., \& Roche, P. F. 1985, MNRAS, 213, 777

Allain, T., Leach, S., \& Sedlmayr, E. 1996a, A\&A, 305, 602

Allain, T., Leach, S., \& Sedlmayr, E. 1996b, A\&A, 305, 616

Allamandola, L. J., Tielens, A. G. G. M., \& Barker, J. R. 1985, ApJ, 290, L25

Allamandola, L. J., Hudgins, D. M., \& Sandford, S. A. 1999, ApJ, $511, \mathrm{~L} 115$

Alonso-Herrero, A., Takagi, T., Baker, A. J., et al. 2004, ApJ, 612, 222 Athey, A., Bregman, J., Bregman, J., Temi, P., \& Sauvage, M. 2002, ApJ, 571, 272

Beck, S. C., Turner, J. L., Langland-Shula, L. E., et al. 2002, AJ, 124

Biviano, A., Sauvage, M., Gallais, P., et al. 1998, The ISOCAM dark current calibration report, Tech. rep., ESA/ISO Data Centre

Brinks, E., \& Klein, U. 1988, MNRAS, 231, 63P

Calzetti, D. 1997, AJ, 113, 162

Cesarsky, C. J., Abergel, A., Agnese, P., et al. 1996a, A\&A, 315, L32

Cesarsky, D., Lequeux, J., Abergel, A., et al. 1996b, A\&A, 315, L309

Chanial, P. 2003, Ph.D. Thesis, Université Paris VII

Claret, A., Dzitko, H., Engelmann, J., \& Starck, J.-L. 1998, Glitch effects in ISOCAM detectors, Tech. rep., ESA/ISO Data Centre

Clayton, G. C., Gordon, K. D., Salama, F., et al. 2003, ApJ, 592, 947

Contursi, A., Lequeux, J., Cesarsky, D., et al. 2000, A\&A, 362, 310

Coulais, A., \& Abergel, A. 2000, A\&AS, 141, 533

Crowther, P. A., Beck, S. C., Willis, A. J., et al. 1999, MNRAS, 304, 654

Dale, D. A., \& Helou, G. 2002, ApJ, 576, 159

Dale, D. A., Silbermann, N. A., Helou, G., et al. 2000, AJ, 120, 583

Dale, D. A., Helou, G., Contursi, A., Silbermann, N. A., \& Kolhatkar, S. 2001, ApJ, 549, 215

Désert, F.-X., Boulanger, F., \& Puget, J. L. 1990, A\&A, 237, 215

Dudley, C. C. 1999, MNRAS, 307, 553

Dudley, C. C., \& Wynn-Williams, C. G. 1997, ApJ, 488, 720

Dufour, R. J., Shields, G. A., \& Talbot, R. J. 1982, ApJ, 252, 461

Dwek, E. 2005, Interstellar dust: what is it, how does it evolve, and what are its observational consequences? AIP Conf. Ser., 488, 103 Engelbracht, C. W., Rieke, M., H., R. G., Kelly, D. M., \& Achtermann, J. M. 1998, ApJ, 505, 639

Engelbracht, C. W., Gordon, K. D., Rieke, G. H., et al. 2005, ApJ, 628, 29

Förster Schreiber, N. M., Sauvage, M., Charmandaris, V., et al. 2003, A\&A, 399, 833

Freedman, W. L., Madore, B. F., Gibson, B. K., et al. 2001, ApJ, 553, 47

Frogel, J., Elias, J., \& Phillips, M. 1982, ApJ, 260, 70

Galliano, F. 2004, Ph.D. Thesis, Université de Paris XI

Galliano, F., \& Dwek, E. 2005, in preparation

Galliano, F., Madden, S. C., Jones, A. P., et al. 2003, A\&A, 407, 159

Galliano, F., Madden, S. C., Jones, A. P., et al. 2005, A\&A, 434, 867

Genzel, R., Lutz, D., Sturm, E., et al. 1998, ApJ, 498, 579 
Gil de Paz, A., Madore, B. F., \& Pevunova, O. 2003, ApJS, 147, 29

Giveon, U., Sternberg, A., Lutz, D., Feuchtgruber, H., \& Pauldrach, A. W. A. 2002, ApJ, 566, 880

Grevesse, N., \& Sauval, A. J. 1998, Space Sci. Rev., 85, 161

Guhathakurta, P., \& Draine, B. T. 1989, ApJ, 345, 230

Guseva, N. G., Izotov, Y. I., \& Thuan, T. X. 2000, ApJ, 531, 776

Heckman, T. M., Robert, C., Leitherer, C., Garnett, D. R., \& van der Rydt, F. 1998, ApJ, 503, 646

Helou, G., Lu, N. Y., Werner, M. W., Malhotra, S., \& Silbermann, N. 2000, ApJ, 532, L21

Houck, J. R., Charmandaris, V., Brandl, B. R., et al. 2004, ApJS, 154, 211

Hunt, L. K., Vanzi, L., \& Thuan, T. X. 2001, A\&A, 377, 66

Hunt, L. K., Giovanardi, C., \& Helou, G. 2002, A\&A, 394, 873

Hunter, D. A., Gallagher, J. S., Rice, W., \& Gillett, F. 1989, ApJ, 336, 152

Hunter, D. A., O’Connell, R. W., \& Gallagher, J. S. 1994, AJ, 108, 84

Israel, F. P. 1988, A\&A, 194, 24

Joblin, C., Boissel, P., Leger, A., D'Hendecourt, L., \& Defourneau, D. 1995, A\&A, 299, 835

Jochims, H., Ruehl, E., Baumgaertel, H., Tobita, S., \& Leach, S. 1994, ApJ, 420, 307

Kessler, M. F., Steinz, J. A., Anderegg, M. E., et al. 1996, A\&A, 315, L27

Kobulnicky, H. A., \& Skillman, E. D. 1997, ApJ, 489, 636

Kobulnicky, H. A., Skillman, E. D., Roy, J., Walsh, J. R., \& Rosa, M. R. 1997, ApJ, 477, 679

Laurent, O., Mirabel, I. F., Charmandaris, V., et al. 2000, A\&A, 359, 887

Leach, S. 1987, Electr. Spectrosc., 41, 427

Léger, A., \& Puget, J. L. 1984, A\&A, 137, L5

LePage, V., Snow, T. P., \& Bierbaum, V. M. 2003, ApJ, 584, 316

Lisenfeld, U., Israel, F. P., Stil, J. M., \& Sievers, A. 2002, A\&A, 382, 860

Lutz, D., Feuchtgruber, H., Genzel, R., et al. 1996, A\&A, 315, L269

Lutz, D., Sturm, E., Genzel, R., et al. 2003, A\&A, 409, 867

Madden, S. C. 2000, New Astron. Rev., 44, 249

Madden, S. C. 2005, in AIP Conf. Proc. 761: The Spectral Energy Distributions of Gas-Rich Galaxies: Confronting Models with Data, 223

Martín-Hernández, N. L., Peeters, E., Morisset, C., et al. 2002a, A\&A, 381, 606

Martín-Hernández, N. L., Vermeij, R., Tielens, A. G. G. M., van der Hulst, J. M., \& Peeters, E. 2002b, A\&A, 389, 286

Mathis, J. S. 1990, ARA\&A, 28, 37

Mattila, F., Lemke, D., Haikala, L. K., et al. 1996, A\&A, 315, L353

Melisse, J. P. M., \& Israel, F. P. 1994, A\&AS, 103, 391

Peeters, E., Martin-Hernandez, N. L., Damour, F., et al. 2002, A\&A, 381,571

Plante, S., \& Sauvage, M. 2002, AJ, 124, 1995

Reach, W. T., Boulanger, F., Contursi, A., \& Lequeux, J. 2000, A\&A, 361,895
Rieke, G. H., Loken, K., Rieke, M. J., \& Tamblyn, P. 1993, ApJ, 412, 99

Rieke, G. H., \& Low, F. J. 1972, ApJ, 176, L95

Rigby, J. R., \& Rieke, G. H. 2004, ApJ, 606, 237

Rigopoulou, D., Kunze, D., Lutz, D., Genzel, R., \& Moorwood, A. F. M. 2002, A\&A, 389, 374

Rigopoulou, D., Spoon, H. W. W., Genzel, R., et al. 1999, A\&A, 118, 2625

Roche, P. F., \& Aitken, D. K. 1985, MNRAS, 213, 789

Roche, P. F., Aitken, D. K., Smith, C. H., \& Ward, M. J. 1991, MNRAS, 248, 606

Roussel, H., Sauvage, M., Vigroux, L., \& Bosma, A. 2001a, A\&A, 372, 427

Roussel, H., Vigroux, L., Bosma, A., et al. 2001b, A\&A, 369, 473

Sauvage, M., \& Thuan, T. X. 1994, ApJ, 429, 153

Sellgren, K., Luan, L., \& Werner, M. W. 1990, ApJ, 359, 384

Siebenmorgen, R., Blommaert, J., Sauvage, M., \& Starck, J.-L. 2000, The ISO Handbook III. CAM - The ISO camera, Tech. rep., ESA/ISO Data Centre

Siebenmorgen, R., Krügel, E., \& Spoon, H. 2004, A\&A, 414, 123

Starck, J. L., Abergel, A., Aussel, H., et al. 1999, A\&AS, 134, 135

Starck, J.-L., Murtagh, F., \& Bijaoui, A. 1998, Image processing and data analysis: the multiscale approach (Cambridge University Press)

Sturm, E., Lutz, D., Tran, D., et al. 2000, A\&A, 358, 481

Sturm, E., Lutz, D., Verma, A., et al. 2002, A\&A, 393, 821

Tacconi-Garman, L., Sturm, E., Lutz, D., Davies, R. L., \& Moorwood, A. 2005, A\&A, 358, 481

Takeuchi, T., Hirashita, H., Ishii, T., Hunt, L., \& Ferrara, A. 2003, MNRAS, 343, 839

Thornley, M. D., Schreiber, N. M. F., Lutz, D., et al. 2000, ApJ, 539, 641

Thuan, T. X., \& Martin, G. E. 1981, ApJ, 247, 823

Thuan, T. X., Sauvage, M., \& Madden, S. 1999, ApJ, 516, 783

Uchida, K. I., Sellgren, K., \& Werner, M. 1998, ApJ, 493, L109

Vacca, W. D., Johnson, K. E., \& Conti, P. S. 2002, AJ, 123, 772

van den Bergh, S. 1999, A\&ARv, 9, 273

Vanzi, L., \& Sauvage, M. 2004, A\&A, 415, 509

Vermeij, R., Peeters, E., Tielens, A. G. G. M., \& van der Hulst, J. M. 2002, A\&A, 382, 1042

Verstraete, L., Pech, C., Moutou, C., et al. 2001, A\&A, 372, 981

Verstraete, L., Puget, J. L., Falgarone, E., et al. 1996, A\&A, 315, L337

Vigroux, L., Aussel, H., Charmandaris, V., et al. 2001, Ap\&SS Suppl., 277, 565

Vogler, A., Madden, S. C., Beck, R., et al. 2005, A\&A, 441, 491

Voit, G. M. 1992, MNRAS, 258, 841

Waller, W. H. 1991, ApJ, 370, 144

Wu, Y., Charmandaris, V., Hao, L., et al. 2005, submitted

Xilouris, E. M., Madden, S. C., Galliano, F., Vigroux, L., \& Sauvage, M. 2004, A\&A 